\title{
Poem-titles in Simonides, Pindar, and Bacchylides
}

\author{
Enrico Emanuele Prodi
}

\section{Introduction}

A volume devoted, among other things, to the lyric paratext invites a discussion of one of its key elements: the titles that accompany the beginning of individual poems in the text of Simonides, Pindar, and Bacchylides. ${ }^{1}$ (I refer to the text rather than to the individual manuscripts for reasons that will become apparent in the next section.) Of the various thresholds to the text anatomised by Gérard Genette, the title is perhaps the most obvious as well as, at least in our day, one of the most pervasive. ${ }^{2}$ Egon von Komorzynski was somewhat over-optimistic when he suggested that 'a comprehensive and thorough history of the book-title could be at the same time a reliable and useful history of intellectual life'; ${ }^{3}$ nonetheless, the study of titles can tell us much about how their creators - be they the authors or the editors of the respective texts - represent such texts to their readership.

\footnotetext{
${ }^{1}$ I am grateful to the editors of the volume and the anonymous reviewer for their thoughtful and valuable comments; they have much improved this paper and bear no responsibility for any remaining mistakes. All translations are mine.

${ }^{2}$ For modern works of titrologie - a term coined by Duchet (1973) - see for instance Levin (1977), the impressive monograph of Hoek (1981), and Genette (1987) 54-97 (1988); references to further literature in Genette (1987) 54 n. 1, Hoek (1981) 339-49. Titles of poetry are investigated by Levenston (1978) and Ferry (1996).

${ }^{3}$ Komorzynski (1903-04) 284.
} 
Unlike the titles of other kinds of compositions, such as tragedies and comedies, which have been investigated several times since the nineteenth century, ${ }^{4}$ the titles of Greek lyric poems have been treated mostly piecemeal: genre by genre, book by book, individual example by individual example. ${ }^{5}$ The main exception isBianca-Jeanette Schröder's volume on poem-titles in Latin poetry, which contains a brief overview of the titles of late archaic Greek lyric, their typology, and their treatment in the manuscripts. ${ }^{6}$ Schröder's discussion gathers a great deal of data and provides many valuable interpretative insights, but a comprehensive analysis of the evidence remains to be attempted. This is therefore the purpose of the present essay.

A Catalogue of all surviving titles from the poetry of Simonides, Pindar, and Bacchylides is provided at pp. 000-000. It will be referred to with bold Arabic figures. Throughout the paper the term 'title' will refer to poem-titles; book-titles will be called such. Admittedly a more neutral term such as 'heading' or indeed 'rubric' would have been more appropriate: we twenty-first-century readers think of titles as the names of certain pieces of literature (Les misérables is called Les misérables much as Victor Hugo is called Victor Hugo), which is not true of most of the titles we shall examine. However, 'title' is the word most commonly used in English-language scholarship to

\footnotetext{
${ }^{4}$ On drama see among others Hippenstiel (1887), Bender (1904), Breitenbach (1908), and most recently Sommerstein (2002). More general treatments of Greek titles are Lohan (1890), Nachmanson (1969), Schmalzriedt (1970); see also many of the papers in Fredouille et al. (1997), concerned with Latin as well as with Greek. Swedish-speaking readers may profit from Zilliacus (1938).

${ }^{5}$ See for instance Schmalzriedt (1970) 26-27 n. 10, Braswell (1988) 55-56, Rutherford (1988) 65 n. 3, (1990) 172, (2001a) 150-52. Beside B.-J. Schröder (1999) 164-68, the only attempt known to me of a general (if cursory) survey of lyric titles is Lohan (1890) 12-19, who, however, neglected those found in the medieval manuscripts of Pindar and (naturally) could not take into account the numerous papyri that were published from the last decade of the nineteenth century onward. Similar titles in Latin poetry are treated extensively by B.-J. Schröder (1999).

${ }^{6}$ B.-J. Schröder (1999) 164-68.
} 
designate them, like the equivalent terms in other languages (titre, titolo, Titel, etc.). Consequently, when a distinction is needed, we shall resort to calling 'titles proper' those that constitute the name of the poem they introduce, that is (for our purposes) titles of dithyrambs such as ”I $\delta \alpha c$ or $\Lambda \varepsilon v \kappa \iota \pi \pi i \delta \varepsilon c(\mathbf{1 0 0}, \mathbf{1 0 2})$. Finally, since our titles were probably systematised (if not devised altogether) in the "standard" Alexandrian editions of the respective poets, ${ }^{7}$ the term 'genuine' in this paper will be used with reference to such editions, not to the earlier time when a poem was composed and first performed.

\section{Transmission}

Titles seem to have been a constant feature of the manuscripts of our three lyricists at least from their "standard" Alexandrian editions onward. ${ }^{8}$ Of the twenty-five published papyri of Simonides lyricus, Pindar, and Bacchylides in which the division between one poem and the next

\footnotetext{
${ }^{7}$ See B.-J. Schröder (1999) 168. The "standard" Pindar is traditionally associated with Aristophanes of

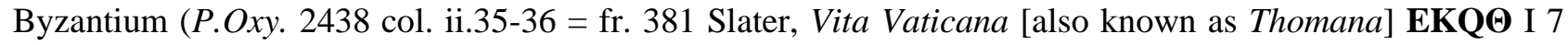
Drachmann); this has been disputed by Slater ad loc., but see D'Alessio (1997) 51-5 and Negri (2004) 16-27 in favour of the traditional attribution. There is no such explicit evidence for Bacchylides, but an argument can be made that the person responsible was Aristarchus, see D'Alessio (1997) 53-54. Likewise no evidence is available for Simonides, but Aristophanes' quotation of the title of one of his poems (12, in fr. 124 Slater) may suggest that he edited his works; similarly Poltera (2008) 12. For our present purposes, what name one attaches to each of these editions matters little, as long as the principle stands of one "standard" edition for each poet, or at least book.

${ }^{8}$ The bibliological aspect of titles in the papyri of our three authors is treated in greater detail in Prodi (2016); this paragraph and the next largely summarise some of its conclusions.
} 
can be recognised, at least nineteen verifiably have titles, ${ }^{9}$ none verifiably has not. In terms of layout, titles come in one of two formats: either inset in the column of writing, variously indented and sometimes spaced away from the verses above or below them (ten to eleven papyri), or written in the margin to the left of the column, with varying degrees of formality, beside or below the marginal signs that marked the end of the previous poem (nine to fourteen). ${ }^{10}$ Although the dataset is too small to allow reliable statistics, the evidence suggests that insetting titles in the column is a later fashion, emerging between the first and the second century AD and becoming prevalent only with the 'severe style' that straddles the second and the third; until some time into the second century, a majority of titles are written in the margins. Marginal titles are sometimes written by the same hand as the text (at least three papyri), sometimes by one or more others (at least four). ${ }^{11}$ Normally these further hands are roughly contemporary to that of the main scribe, but there is one important exception: Bacchylides’ P, where the title of fr. ${ }^{*} 20 \mathrm{C}$ Maehler (106) seems to have been added a long time after the text was written. ${ }^{12}$

These data do not necessarily indicate that intitulation is a later practice that crept into the manuscripts, first added in the margins by readers' hands, then slowly incorporated into the text. Among our three authors there is not one certain instance of a papyrus that lacked titles altogether; even $\mathbf{P}$ was furnished with titles eventually. Of course, given that $\mathbf{P}$ had no titles originally and that our papyrus evidence is heavily concentrated in the second and third centuries AD, it cannot be excluded that (some) early manuscripts of the Alexandrian vulgate of our authors may have had no

\footnotetext{
${ }^{9}$ A possible twentieth is $\Pi^{10}$, where the argument for the presence of the title of fr. 94b Snell-Maehler $(* 76)$ relies on a single trace on the edge of a lacuna and is therefore doubtful: see Prodi (2016) 1144-1145.

${ }^{10}$ For a table of the evidence for the different formats and hands see Prodi (2016) 1176; for their distribution over time, ibid. 1179.

${ }^{11}$ The inset titles are all written by the same hand as the text, as one would expect.

${ }^{12}$ The text dates from the late first century BC or early first AD, as shown by Cavallo (1974) 36 and n. 20; the hand of the title belongs in the second or even third AD, see Prodi (2016) 1146.
} 
titles by design. The Hellenistic period was one of experimentation and variety in matters bibliological no less than in others, and not all paratextual features need to have been as standard then as they would go on to be. Two important early manuscripts of 'choral' lyric $-\mathbf{P}$ itself and P.Oxy. 1790 (Ibycus' 'Ode to Polykrates', PMGF S151) - lack the marginal markers of metrical articulation that almost all our other papyri have. Even such a (later) regular feature of the literary book-roll as the end-title only becomes generalised at the turn from the Hellenistic to the Roman period, at least in Egypt. ${ }^{13}$ Nevertheless, titles in at least one book certainly had an early origin: Bacchylides' Dithyrambs were arranged alphabetically by title, indicating that their titles go back to (at least) the Alexandrian edition itself, in which that order of the poems was established. ${ }^{14}$ Likewise, if Simonides' Europa was one of his Dithyrambs (see 12 with $n$.), the citation of its title by Aristophanes of Byzantium suggests that it goes back at least as far as his (?) edition of that

${ }^{13}$ See Schironi (2010) 25-28, (2016) 85. In Herculaneum some $90 \%$ of the rolls whose final portion is preserved (mostly dating to the first century BC) have end-titles: Del Mastro (2014) 7.

${ }^{14}$ B.-J. Schröder (1999) 164-65, Rutherford (2001) 150. The same will apply to any other books that were similarly organised, even though it is unclear how many were. Such an arrangement has been suggested for Simonides' Epinicians for equestrian victors (or a section thereof: D'Alessio [1997] 53 n. 175) and Paeans (Poltera [2008] 169-70) as well as Pindar's Prosodia (D'Alessio [2004] 114) and a section of his Paeans (Rutherford [1995] 49 n. 24, D’Alessio [1997] 31 n. 45 and [2001] 84). I have expressed some doubts regarding Pindar's Prosodia in Prodi (2013) 56-58; as regards Simonides, the apparently identical format of 2 and 4 - which shows that $P M G 511=$ fr. 7 Poltera was not necessarily the first poem in a sub-section weakens the argument on the Epinicians for equestrian victors somewhat, but does not disprove its conclusions. I do not know on what evidence Poltera (2008) 12 claims that Simonides' Dithyrambs were also alphabetically arranged. Unable to resist the temptation to add some speculation of my own, I have suggested that also Pindar's Partheneia, or a section thereof, may have been arranged alphabetically: Prodi (2014) 10405. 
book. ${ }^{15}$ While it is possible that dithyrambic titles, being the only titles 'proper' in our dataset, had a special status and therefore were canonised earlier than the rest, ${ }^{16}$ nothing in our evidence requires this conclusion. The question why it was relatively common for titles to be written by further hands remains open. One may imagine a fashion of sorts - even though not one associated with a particular script or layout - or a belief that only the poet's words were the main scribe's province, while paratextual material such as titles ought to be left to the diorthotes.

At all events, titles were normally transmitted together with the poems they accompanied. In at least two of the four papyri where titles are written by a second hand, these are in the corrector's hand, not in that of a subsequent annotator. ${ }^{17}$ The Pindaric scholia - later in form but largely Hellenistic and early Imperial in content - begin their exposition of each ode with a headnote (inscriptio in Drachmann's Latin) which is, for all intents and purposes, a scholion to its title. Occasionally a headnote actually comments on the title as if it were part of the text: for instance those of Olympians 3 and 11, Pythian 10, and Isthmian $3 .{ }^{18}$ Similarly, the odd position of a scholion to Pindar's Paean 6(b) in $\Pi^{4}$ - written beside the second line of the piece rather than the first ${ }^{19}-$ is

\footnotetext{
${ }^{15}$ See n. ooo above.
}

${ }^{16}$ On the possibility that some of them may predate their Alexandrian canonisation see pp. ooo below.

${ }^{17} \Pi^{4}$ of Pindar and A of Bacchylides; a third, doubtful case is $\Pi^{9}$, where however Houston (2014) 169 suggests ascribing the title to the annotator. $\mathbf{A}$ is a particularly obvious case, in that except for the corrections it was not annotated at all (more on which at pp. ooo below). Another papyrus which may have had titles but apparently no annotations is $\Pi^{10}$, on which see $\mathrm{n}$. ooo above.

${ }^{18}$ Inscrr. A Ol. 3, I 105 Drachmann; BCDEQ Ol. 11, I 342 Drachmann; BDEGQ Pyth. 10, II 241-42 Drachmann; BD Isthm. 3, III 223 Drachmann. See B.-J. Schröder (1999) 167.

${ }^{19}$ Schol. 124, 304 Rutherford. This is not the place to discuss in detail the status of the "third triad" of Pae. 6 $=$ Pae. 6(b) following the discovery of its title and the correct supplementation of schol. 124 by Rutherford and D'Alessio ap. Rutherford (1997a) 3-8. Secure data are that the piece - I use this term so as not to prejudge the complex question of whether it was ever an independent poem - appeared both in the Paeans 
best understood as aligned with the word $\pi \rho \circ[\mathrm{o}] \delta \mathrm{r}[\mathrm{o}] \nu$ in the title on the other side of the column (65), which is the target of the explanation. Although Pindar's Nemeans from the eighth onward and all of the Isthmians have no titles in the surviving medieval manuscripts, some of their titles are preserved elsewhere. Those of Nemean 10 and Isthmian 4 partly survive on papyrus $(\mathbf{4 7}, \mathbf{5 1}) .{ }^{20}$ That of Ishmian 3 is referred to by the scholia, as we have just seen $(* \mathbf{5 0})$; so is that of Isthmian 1 $(* \mathbf{4 8}) .{ }^{21}$ The title of Isthmian 5 is implied by two of its inscriptions or headnotes $(* \mathbf{5 2}$ with $n$.), and the same is probably true of that of Nemean $8(* 46$ with $n.){ }^{22}$ So, whenever independent evidence is available, the absence of a title from the medieval manuscripts of Pindar can be shown to be a later loss. The other odes whose titles are not preserved probably lost them in transmission, too.

To summarise, the evidence at our disposal supports two related conclusions, at least on a general level. Firstly, in most manuscripts of Pindar, Bacchylides, and Simonides individual poems were furnished with titles as a matter of routine. Secondly, in the respective "canonical" editions every ode had a title, which it tended to retain from copy to copy, at least in antiquity, unless error or negligence intervened. ${ }^{23}$ And it is to antiquity that this discussion will be confined; the Byzantine manuscripts will only be used as sources for their Hellenistic and Roman ancestors.

and in the Prosodia, and that it was accompanied by a title of its own in the former book too (65); the evidence further suggests that at least it was treated as an independent poem in the Alexandrian edition of Pindar, not only in the Prosodia but also in the Paeans. My discussion in this paper assumes this conclusion, as well as the corollary that the "first two triads" of Pae. $6=P a e .6$ (a) were also treated as a self-standing composition.

${ }^{20}$ P.Oxy. 5043 fr. 15 (Nem. 10), $\Pi^{25}$ A fr. 2(a) (Isth. 4).

${ }^{21}$ Inscr. a BD, III 196 Drachmann.

${ }^{22}$ Inscrr. b BD, c B Isthm. 5, III 241 Drachmann; BD Nem. 8, III 140 Drachmann.

${ }^{23}$ Irigoin (1952) lists the titles that, by stemmatic inference from the Byzantine manuscripts, go back at least to the commented edition of Pindar's Epinicians that was probably compiled in the second century AD (101), to the archetype of the Vatican recension in the early fifth (108-09), and to the nearest common 
Despite the variation that the medieval manuscripts sometimes display in matters of detail, titles appear to have been transmitted fairly consistently. Even the medieval manuscripts tend to agree with one another in the essentials. Overlap between papyri and medieval manuscripts is scarce (but see nonetheless $\mathbf{1 4}$ and 37), and overlap between different papyri it is practically nonexistent, but the overall coherence in terms of pattern - which we shall examine shortly - does not suggest that titles were in a state of generalised chaos. A scholiast remarks that the title of Pythian

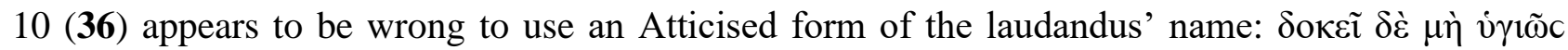

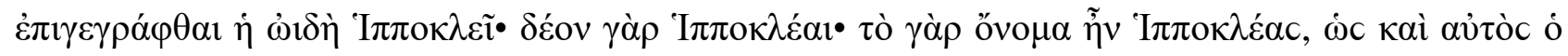

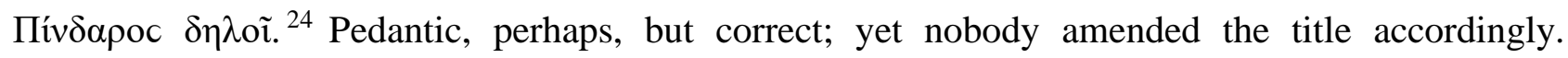
Apparent exceptions are not entirely such. The title of Pythian 11 in P.Oxy. 5042 (37) is likely to have been incomplete; the same may be true of that of Partheneion 2 in $\Pi^{10}(* 76)$, if it was present at all. ${ }^{25}$ But partial omission is not equivalent to outright invention or wilful alteration. As far as one can judge, the process that took place across the Hellenistic and Roman period after the "standard" editions of our three poets were established is largely one of direct transmission, not of active recreation at each copyist's or reader's whim. Although titles were not always complete in every manuscript (but they were far more frequently than not), it is plausible that what was written was indeed, usually, genuine. ${ }^{26}$

ancestor of the shortened Vatican recension in the late tenth (132). Nothing in his inquiry contradicts the present argument that the titles are all in fact earlier, ultimately going back to Alexandria.

24 "It seems that the ode is not titled "to Hippokles" properly: it should have been "to Hippokleas", for the name is Hippokleas, as Pindar himself makes clear' (inscr. BDEGQ, II 241-42 Drachmann). On the (partial) Atticisation of personal names in our titles see pp. 000 below.

${ }^{25}$ See also the discussion of the titles in Bacchylides' A just below.

${ }^{26}$ Once again, 'genuine' from a purely text-critical and text-historical perspective - for our purposes, 'faithfully representing the respective Alexandrian original' - with no implication of veracity. On the reliability or otherwise of the information provided by titles see pp. 000 below. 
One possible problem is the London Bacchylides, $\mathbf{A} .^{27}$ Its text was corrected four times: by the main scribe $\left(\mathbf{A}^{\mathbf{1}}\right)$ and by three correctors, conventionally known as $\mathbf{A}^{\mathbf{2}}, \mathbf{A}^{\mathbf{3}}, \mathbf{A}^{\mathbf{4}} \cdot{ }^{28}$ The main scribe wrote no titles, at least in the surviving portions of the manuscript. $\mathbf{A}^{\mathbf{2}}$ supplied those of odes 2, 19, 20, and perhaps, mistakenly, $7{ }^{29} \mathbf{A}^{\mathbf{3}}$ took care of odes $3,4,6-15,17$, and 18 (see 87-95, 97-101). Ode 5 was left untitled, probably by mistake. There is no obvious explanation for why it took two hands to fill in the titles (perhaps like $\Pi^{4}$ of Pindar's Paeans), ${ }^{30}$ and as the question has no obvious impact on our discussion, it will be left aside. But a more important issue are the two correctors' sources. $\mathbf{A}^{\mathbf{3}}$ certainly corrected by collation, whether of the exemplar from which $\mathbf{A}$ was copied or of a second manuscript, ${ }^{31}$ but this may not be true of $\mathbf{A}^{\mathbf{2}}$. The latter's corrections to the poetic text

\footnotetext{
${ }^{27}$ With reference to A, I use the singular 'papyrus' or 'manuscript' for the sake of convenience, without prejudice to the question whether it was one book-roll or two: see Prodi (2016) 1159 n. 73.
}

${ }^{28}$ See Kenyon (1897) xix-xx, liii, and Jebb (1905) 132-35.

${ }^{29}$ According to Kenyon (1897) 65, the title of 7 (90) added by $\mathbf{A}^{\mathbf{3}}$ is 'written over three washed-out lines, perhaps a repetition of the full title as given at the beginning of the previous poem'; he did not speculate that the earlier writing was by $\mathbf{A}^{\mathbf{2}}$, as Snell (19345) 11* went on to suggest. Snell's reconstruction of the deletion and replacement of the title of 7, if correct, is the only evidence that $\mathbf{A}^{\mathbf{3}}$ corrected the manuscript after $\mathbf{A}^{\mathbf{2}}$ : if he is mistaken - for instance, if $\mathbf{A}^{\mathbf{3}}$ himself had miswritten the title that he then deleted and corrected - the reverse order is equally possible. It is unclear what importance a reversal of the two correctors may have for our discussion, but the more limited role played by $\mathbf{A}^{2}$ would be easily explained if most of the corrections had already been made by $\mathbf{A}^{3}$.

${ }^{30}$ According to Grenfell and Hunt (1908) 15 , the two titles found on that manuscript $(\mathbf{6 4}, \mathbf{6 5})$ are in the same hand; according to Rutherford (1997a) 4, 20, they are not. Given how little text survives, the question is hard to settle.

${ }^{31}$ Supplementation by $\mathbf{A}^{\mathbf{3}}$ of lines omitted by $\mathbf{A}$ proves that he had access to a manuscript other than $\mathbf{A}$ itself, as Kenyon (1897) xviii remarks; the false corrections he made to several authentic readings of $\mathbf{A}$ suggest that his exemplar was different from that of $\mathbf{A}$, but other hypotheses can be entertained, see Prodi (2016) 1182. 
are all simple enough to have been conjectural, even though there is no proof that they were. The implications for the titles are obvious: if $\mathbf{A}^{\mathbf{2}}$ relied on nothing but $\mathbf{A}$ and his own ingenuity, it follows necessarily that the titles he wrote are conjectural too. Bruno Snell argues precisely this, on the grounds that $\mathbf{A}^{2}$ could easily have extracted the necessary information from the text of the poems. $^{32}$

This is of particular import for the Dithyrambs. The two whose title was supplied by $\mathbf{A}^{\mathbf{2}}, 19$ and 20, are the only ones where the title 'proper' is followed by the indication of the community for whom the poem was composed $(\mathbf{1 0 0}, \mathbf{1 0 1})$, which is not found in those written by $\mathbf{A}^{\mathbf{3}}$. What are we to make of this? It seems uneconomical to suggest that $\mathbf{A}^{\mathbf{2}}$ and $\mathbf{A}^{\mathbf{3}}$ used the same manuscript, whether the exemplar of $\mathbf{A}$ or another copy, but $\mathbf{A}^{\mathbf{2}}$ transcribed the titles faithfully while $\mathbf{A}^{\mathbf{3}}$ ignored the second component of each (and yet preserved the double titles of $\mathbf{9 7}$ and $\mathbf{9 8}$ ). ${ }^{33}$ To suppose that $\mathbf{A}^{\mathbf{2}}$ and $\mathbf{A}^{\mathbf{3}}$ availed themselves of different exemplars, one with longer and one with shorter titles, merely pushes the conundrum up the stemma. Moreover, if PSI 1811 does represent Bacchylides' Dithyrambs, ${ }^{34}$ there is another, independent example of a title without an indication of the commissioning community (102). If this was a mere omission, it was suspiciously widespread. On the other hand, it would also be strange - albeit certainly not impossible - for $\mathbf{A}^{\mathbf{2}}$ to have conjectured titles beyond the attested format. The Alexandrian Pindar may have contributed to the error by providing misleading parallels (75), but titling patterns in the two authors do tend to correspond, as we shall see. At least the titles 'proper' of $\mathbf{1 0 0}$ and $\mathbf{1 0 1}$ must be correct, in that they

As we saw, the mere fact that the main scribe did not copy titles is no evidence that there were none in his antigraph.

${ }^{32}$ Snell $\left(1934^{5}\right) 10^{*}$.

${ }^{33} \mathbf{A}^{3}$ also omitted the specification $\pi \alpha 1 \delta$ í in $\mathbf{9 0}$ (see $n$.), but copied it correctly in $\mathbf{9 3}$. It is hard, though possible, to argue that he was prone to omitting seemingly less relevant details generally.

${ }^{34}$ First attributed to Bacchylides by Vogliano (1932) 172; this ascription is generally accepted, despite the attempt by Davison (1934) to suggest Simonides instead. See most recently Hadjimichael (2014) 81-85. 
respect the alphabetic arrangement. Furthermore, a title may be more memorable than other elements of the text: if $\mathbf{A}^{\mathbf{2}}$ knew his Bacchylides, he might have remembered, rather than conjectured, the few titles he filled in even if he did not have a copy at hand against which to collate the text on a large scale. But it is also possible that $\mathbf{A}$ genuinely reflects the condition of titles in Bacchylides' Dithyrambs - some with datives, some without - and that the coincidence between typologies and hands is only that - a coincidence.

We have seen how our titles probably go back to the "standard" Alexandrian editions of our three poets. In the last chapter we shall examine some cases in which a title (or part of one) may be earlier than those editions. It is also likely that, in the two and a half centuries before the activity of the Alexandrian philologists, odes were at least occasionally referred to with something resembling our titles (we have a few examples thanks to Athenaeus), ${ }^{35}$ whether or not these accompanied them in their written form too. However, titles as we have them seem to be largely an Alexandrian creation, or at least systematisation. The consistency of their format within books and genres as well as across the three authors betrays the hand of the editor, if not of the librarian; that they may have arisen independently in all of the (presumably) many streams of tradition that brought the poetry of Simonides, Pindar, and Bacchylides from all over the Greek-speaking world to Alexandria defies belief. Their peculiar semi-Attic veneer, with its distinctive combination of inconsistently Atticised

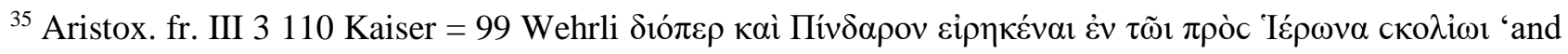
Pindar said in the skolion to Hieron'; Heraclid. Pont. fr. 39 Schütrumpf $=55$ Wehrli $=$ Пív $\delta \alpha \rho o c ~ \pi \alpha \rho \alpha ı \tilde{\omega} v$

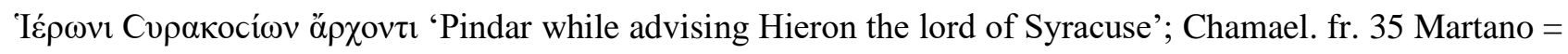

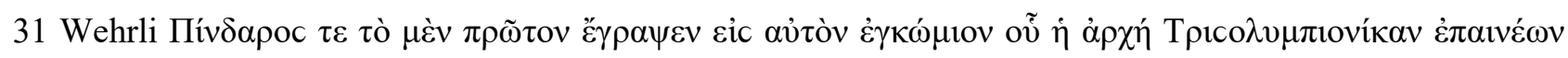

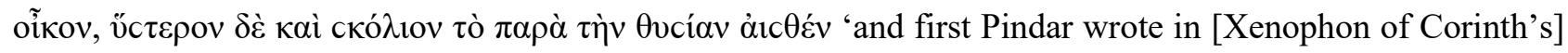
honour the enkomion that begins "In praising a house with three Olympic victories" [Ol. 13], and later also

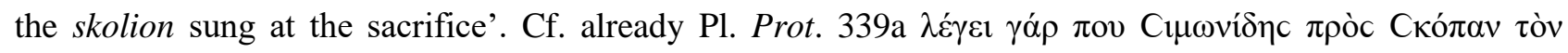

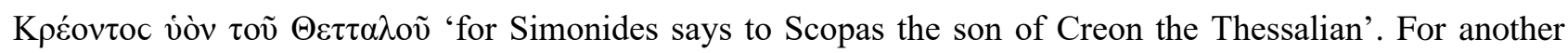
possible hint see n. 000 below.
} 
endings and retained non-Attic vowels in the stem (see $\mathbf{1 8}, \mathbf{2 2}, \mathbf{3 0}, \mathbf{3 6}, \mathbf{4 1}, \mathbf{4 2}, * \mathbf{5 2}, * \mathbf{5 3}$ ), also tells against direct provenance from the poet's own estate or from the different localities (most of them outside the Attic-speaking sphere) for which the poems had been composed. Given the important role that Athenian audiences, and indeed readerships, probably played in the transmission of Pindar's poetry between the fifth and the third century, ${ }^{36}$ it is tempting to wonder whether the Alexandrian system of titles may have been built upon an Attic antecedent, or at least upon Attic examples. But the scarce evidence for pre-Alexandrian titles complicates any attempt to assess of this question, which is best left aside now.

\section{Catalogue}

The Catalogue aims to include all attested poem-titles in the poetry of Simonides, Pindar, and Bacchylides, both those preserved directly by papyri or (in Pindar's case) medieval manuscripts of the poetry itself, and titles quoted by other sources with varying degrees of fidelity. When there is a reasonable possibility that a title is not quoted verbatim or in its entirety, the relevant entry is marked with an asterisk. In several cases one may doubt whether the source was actually referring to the title or rather giving an unrelated piece of information on the poem, but I have chosen to err on the side of inclusiveness; likewise with the information given by the headnotes in the Pindaric scholia, whose derivation from the respective titles is not beyond doubt. As a rule, I have not tampered with transmitted material so as to produce a neater picture. Nonetheless, some patently tralaticious information that leaked into the titles from the scholia in some Byzantine manuscripts of Pindar's Epinicians is relegated to the apparatus for the sake of clarity. ${ }^{37}$ Manuscript readings for

\footnotetext{
${ }^{36}$ See Hadjimichael, this volume pp. 000-000. Compare the argument in D'Alessio (2016) 80-84 for an Athenian tradition of sympotic performances of Bacchylides' skolia.

${ }^{37}$ See Irigoin (1952) 108-09.
} 
the titles of Pindar's Epinicians are taken from Turyn (1944), (1948), retaining his sigla but

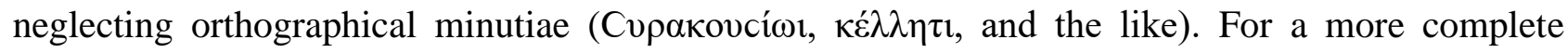
overview of the textual transmission, readers should refer, as always, to Mommsen (1864). I have examined all the papyri either personally or on good photographs; reference to them is made with Snell and Maehler's sigla when available, otherwise with the respective publication numbers. Readings and supplements are due to the respective editores principes unless otherwise stated.

\section{Simonides}

\section{Epinicia}

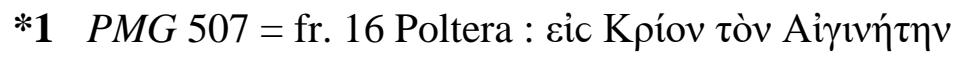

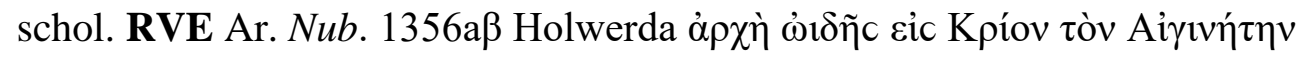

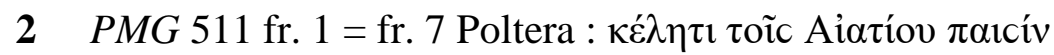

P.Oxy. 2431 fr. 1

*3 PMG 514 = fr. 3 Poltera : cic 'O

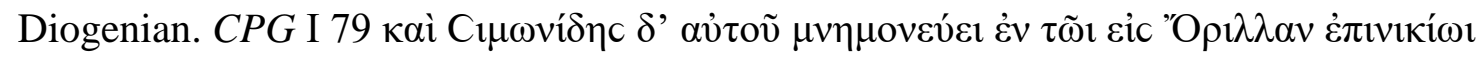

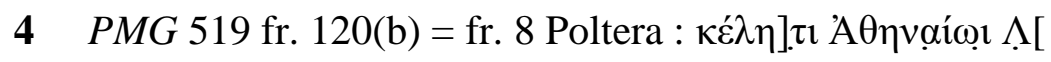

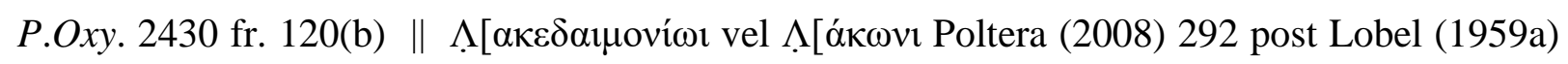
81

Paeanes

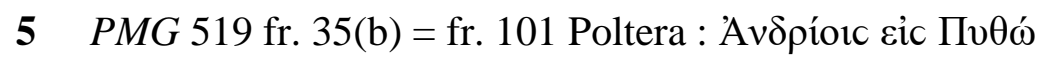

P.Oxy. 2430 fr. 35 
Hymni

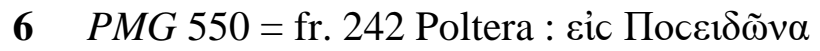

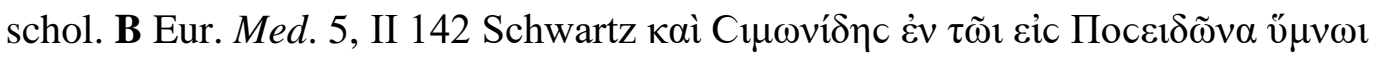

\section{Threni}

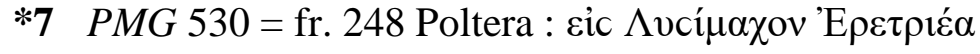

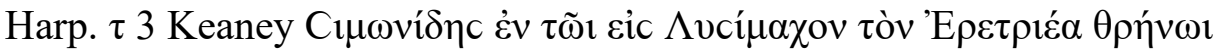

\section{Dithyrambi}

$8 \quad P M G 539=$ fr. ${ }^{\circ} 351$ Poltera : Mé $\mu \nu \omega v$

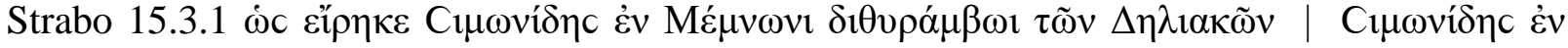

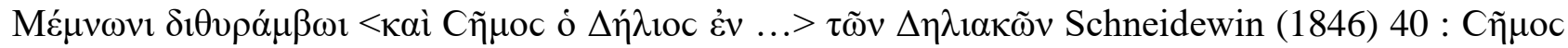

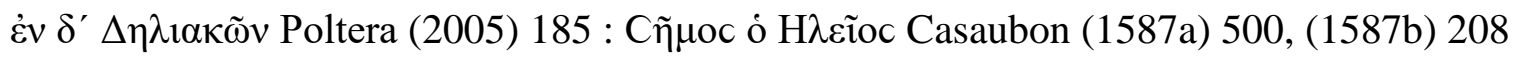

Incerta

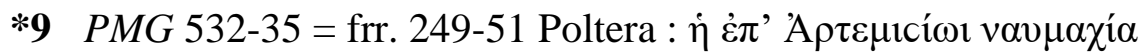

Prisc. gramm. III 428 Keil Simonides in Epartemisio Naumachia | cf. Sud. c 439 Adler אaì $\dot{\eta}$

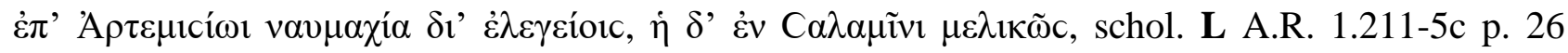
Wendel

*10 PMG $536=$ fr. 252 Poltera : $\dot{\eta} \dot{\varepsilon} v$ C $\alpha \lambda \alpha \mu \tilde{\imath} v \imath<v \alpha v \mu \alpha \chi i ́ \alpha>$

Sud. c 439 Adler cit. ad *9

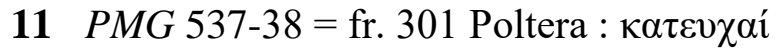

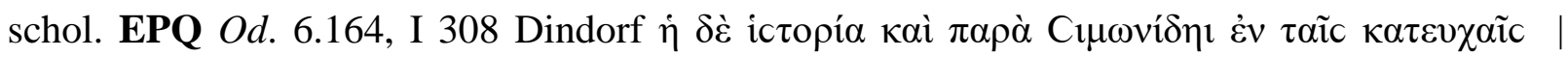

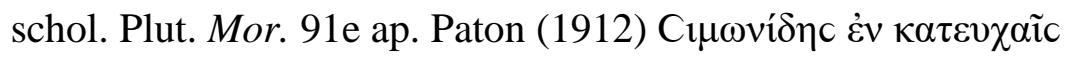

$12 P M G 562$ = fr. 253 Poltera : Eủ

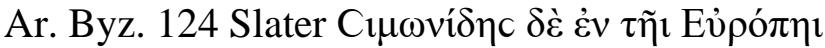




\section{Pindarus}

Epinicia

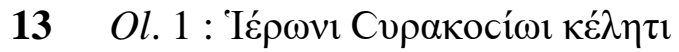

\section{LEHUCN}

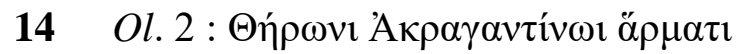

ABLEGHCN $\Pi^{1} \| \Theta \eta ́ p \omega v \imath ~ \alpha \kappa \rho \alpha\left[\Pi^{1}\right.$

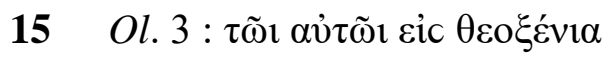

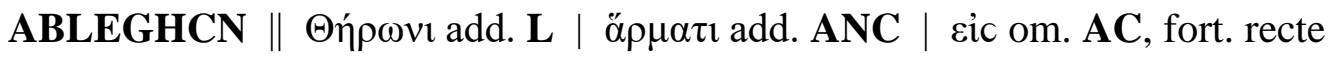

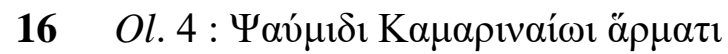

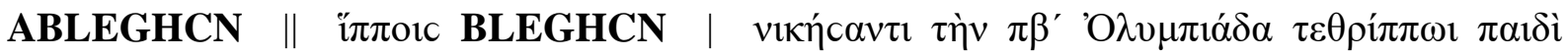

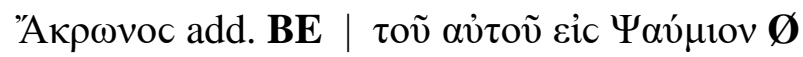

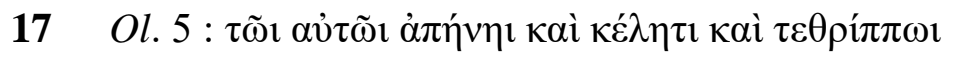

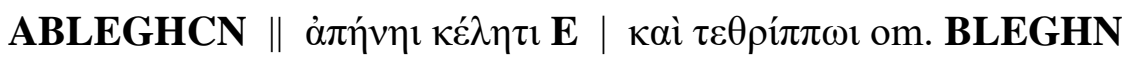

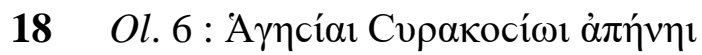

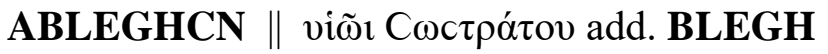

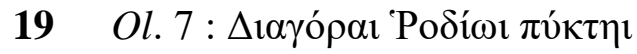

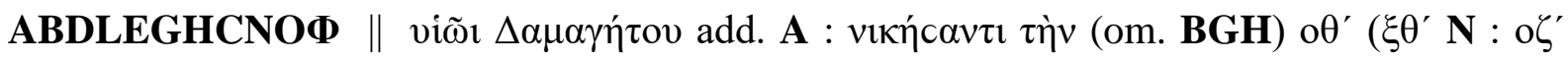

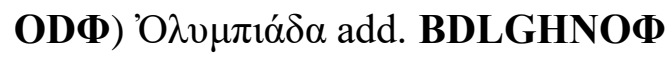

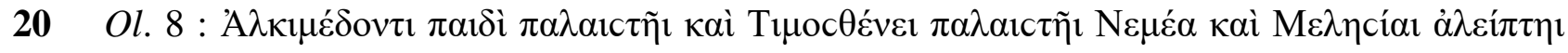

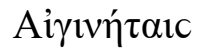

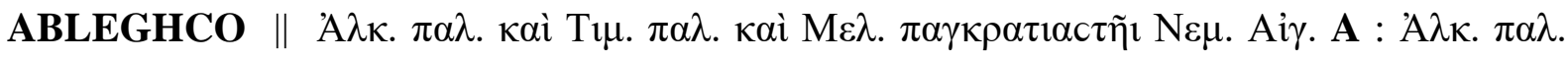

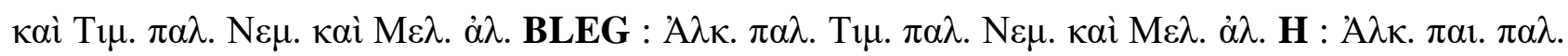

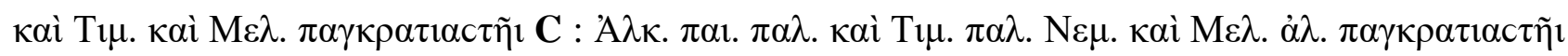

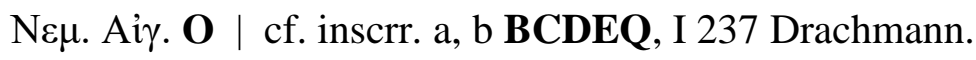




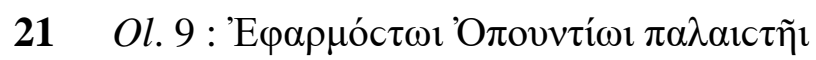

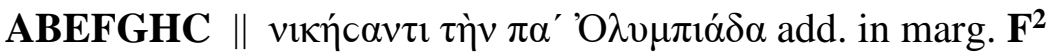

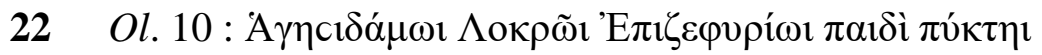

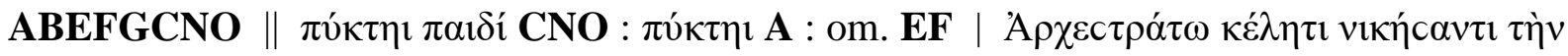
$\pi \beta^{\prime}{ }^{\prime} \mathrm{O} \lambda v \mu \pi \mathrm{i} \alpha \dot{\delta} \alpha$ add. $\mathbf{F}^{2}$

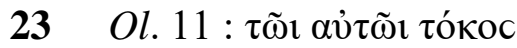

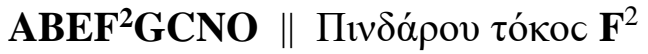

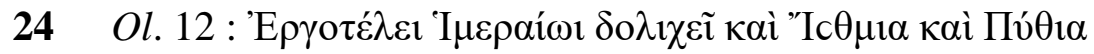

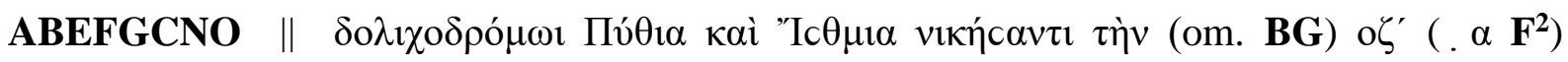
'O $\lambda v \mu \pi \iota^{\alpha} \delta \alpha$ BEFG : $\delta 0 \lambda \imath \chi \varepsilon \tilde{\imath}$ CNO

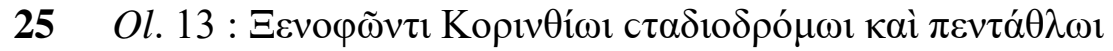

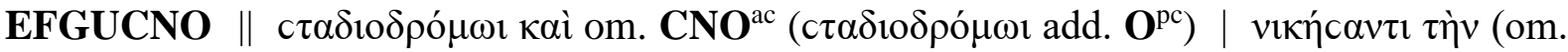

EG) $o \theta^{\prime}\left(\pi \theta^{\prime} \mathbf{F}^{2}\right){ }^{\prime} \mathrm{O} \lambda v \mu \pi \alpha^{\prime} \delta \alpha$ add. EFGU

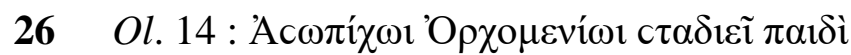

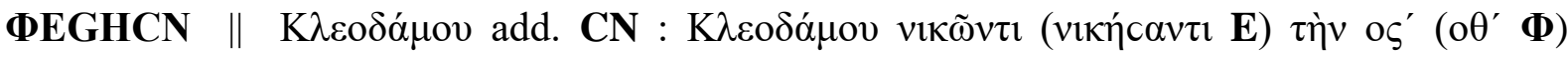
'O $\lambda v \mu \pi i \alpha ́ \delta \alpha$ add. ФEGH

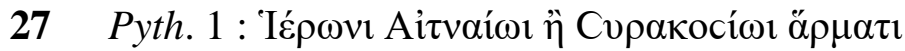

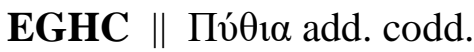

28 Pyth. 2 : $\tau \tilde{\omega} \iota \alpha \jmath ่ \tau \tilde{\iota}$

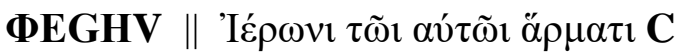

29 Pyth. 3 : $\tau \tilde{\omega} \iota \alpha \jmath ่ \tau \tilde{\omega} 1 \kappa \varepsilon ́ \lambda \eta \tau \imath$

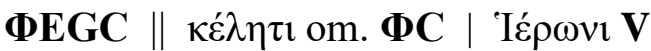

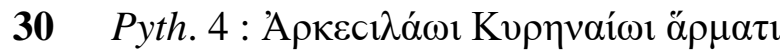

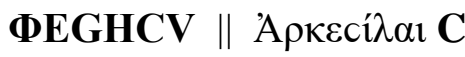

$31 \quad$ Pyth. 5 : $\tau \tilde{\omega} 1 \alpha \tilde{\tau} \tau \tilde{\omega} 1$

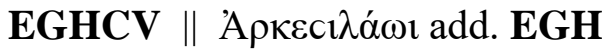




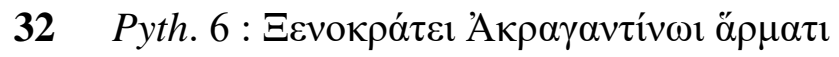

\section{EGHV $^{2}$}

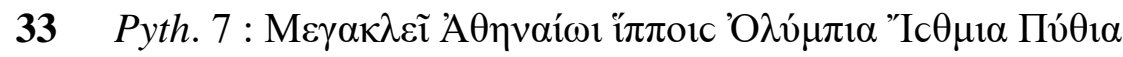

EGHV $\|$ Пv́$\theta \imath \alpha$ om. E, del. Drachmann II 200

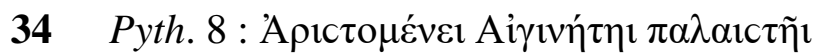

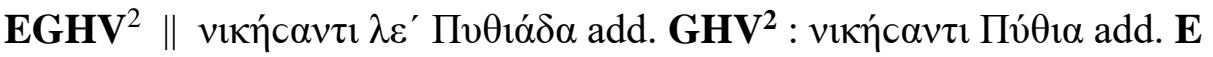

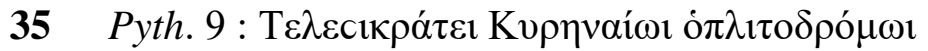

\section{EGHV $^{2}$}

$36 P y t h .10$ : $1 \pi \pi 0 \kappa \lambda \varepsilon \tilde{\imath} \Theta \varepsilon c c \alpha \lambda \tilde{\omega} 1 \pi \alpha \imath \delta i ̀ ~ \delta 1 \alpha v \lambda o \delta \rho o ́ \mu \omega \imath$

EFGHV $^{2} \|$ bis $\mathbf{H}$

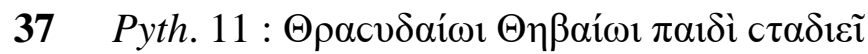

EFGHV P.Oxy. $5042 \| \quad c \tau \alpha \delta \imath \delta \rho o ́ \mu \omega \imath \mathbf{G}:$ om. F $\mathbf{F}^{\mathrm{pc}} \mid$ solum $\Theta \rho[\alpha] c v \delta[\alpha i ́ \omega \imath$ fort. habebat P.Oxy. 5042

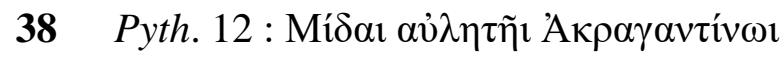

\section{EFGHV $^{2}$}

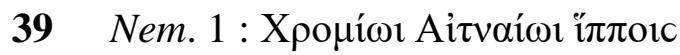

$\mathbf{U} \mathbf{V}^{2}$

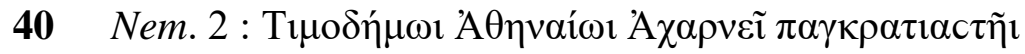

DTU $\| \pi \alpha \gamma \kappa \rho \alpha \tau \iota \alpha c \tau \tilde{\eta} \iota$ Moschopulus : $\pi \alpha \gamma \kappa \rho \alpha \tau \varepsilon \tilde{\imath} \mathbf{D}: \pi \alpha \gamma \kappa \rho \alpha ́ \tau \iota$ T TU

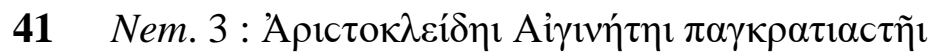

D

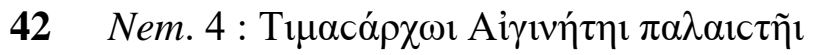

D

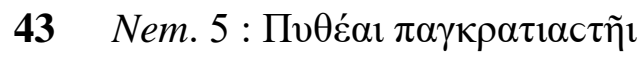

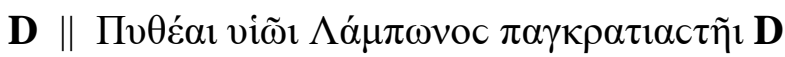

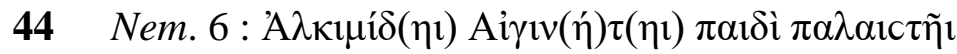




\section{D}

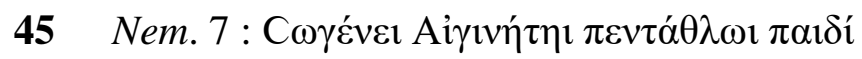

D

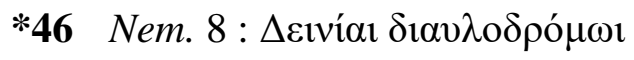

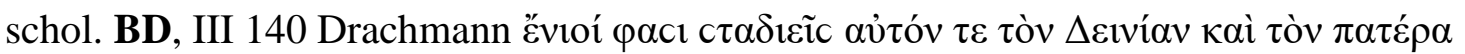

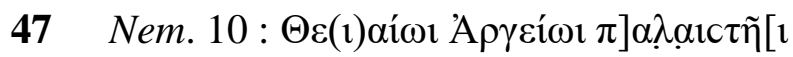

P.Oxy. 5043 fr. 15

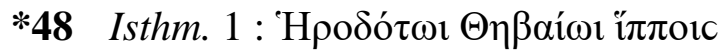

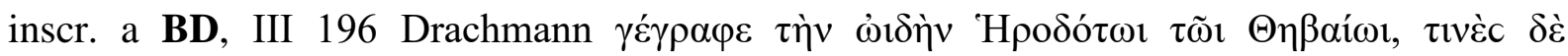

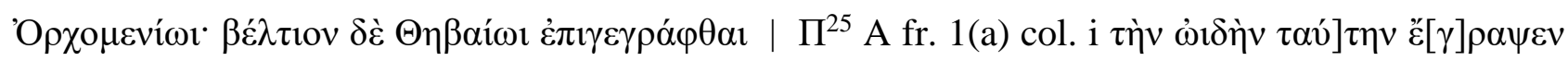

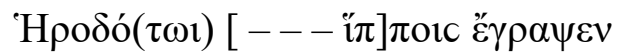

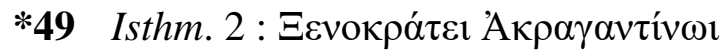

inscr. a BD, III 212 Drachmann oṽ

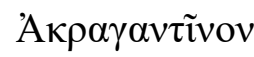

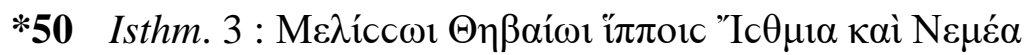

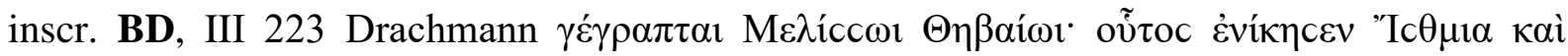

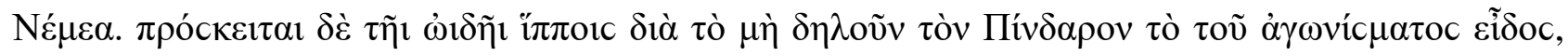

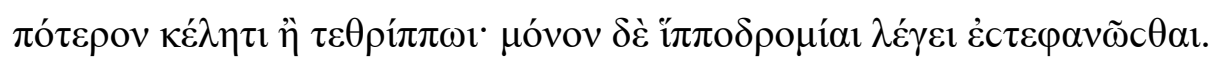

$51 \quad$ Isthm. $4: \tau] \tilde{\omega} \iota \alpha \jmath ่ \tau[\tilde{\omega} \iota$

$\Pi^{25}$ A fr. 2(a)

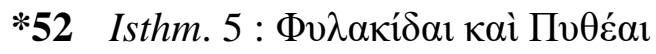

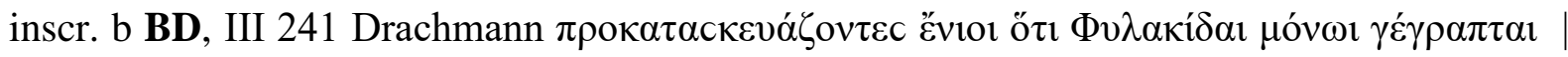

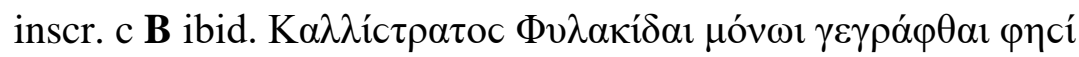

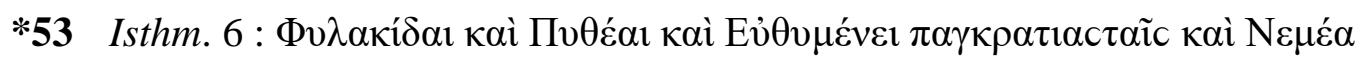

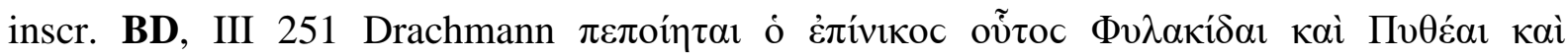

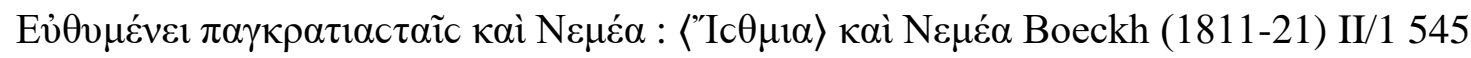




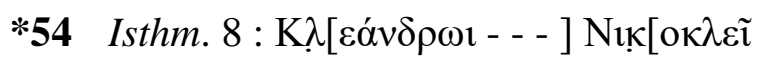

$\Pi^{25}$ A fr. 4(a)

55 ]. $\varepsilon v .[$

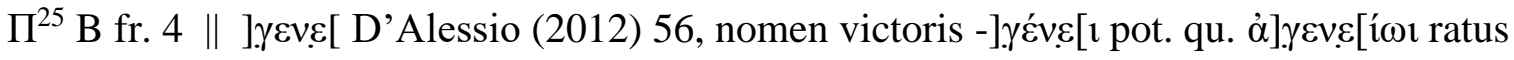

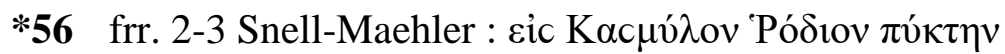

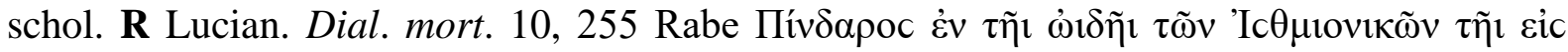

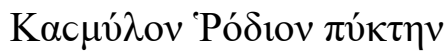

*57 fr. 4 Snell-Maehler : Meıó́aı

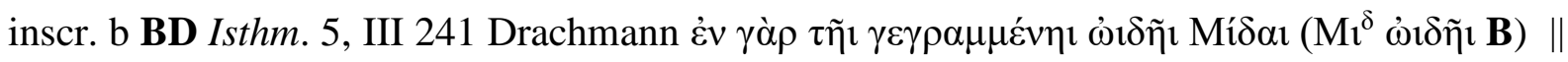

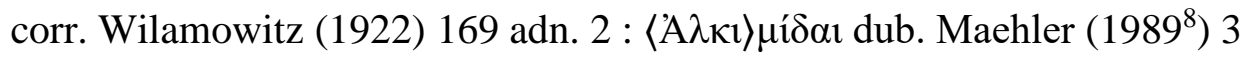

58 fr. 6a Snell-Maehler : ]

$\Pi^{25}$ B fr. 14

59 fr. 6a(i)-(1) Snell-Maehler : ] $\pi \alpha \gamma \kappa \rho \alpha \tau ı \alpha c \tau \alpha i ̃ c ~ ' E \lambda \lambda \omega ́ \tau \imath \alpha$

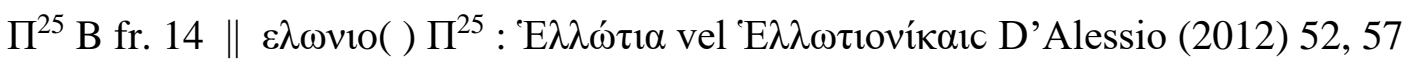

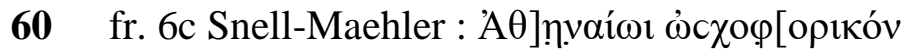

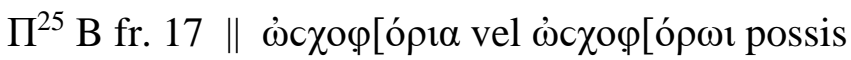

Hymni

*61 fr. 36 Snell-Maehler : A $\mu \mu \omega v i ́ o r c ~ ’ A \mu \mu \omega v \imath ~$

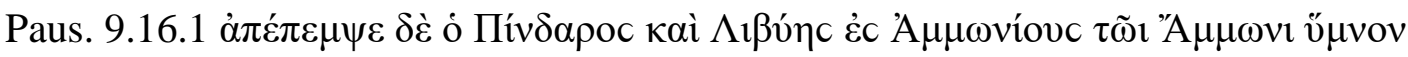

*62 fr. 37 Snell-Maehler : cic $\Delta \eta \dot{\eta \eta \eta} \tau \rho \alpha$

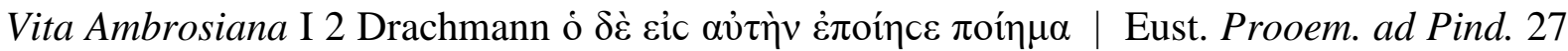

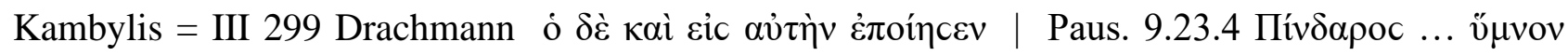

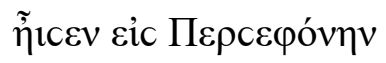




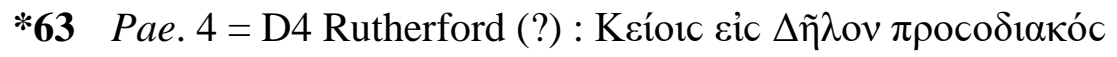

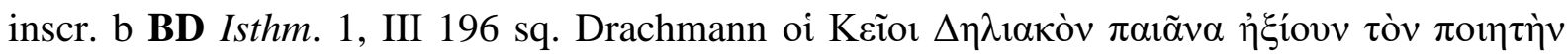

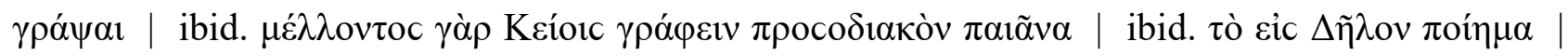

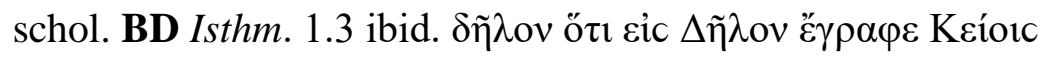

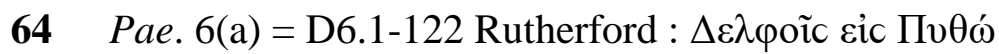

$\Pi^{4}$ col. xxii

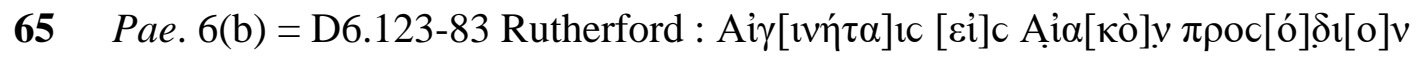

$\Pi^{4}$ col. xxx $\|$ suppl. Rutherford (1997a) 3-6

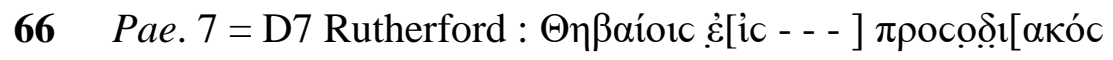

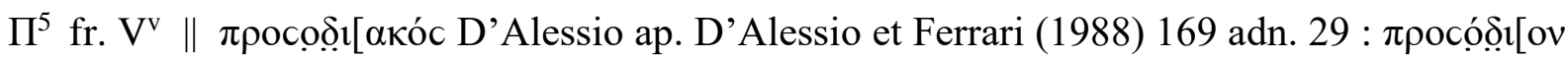
Rutherford (1997a) 5 adn. 17

67 Pae. $7 \mathrm{~b}=\mathrm{C} 2$ Rutherford : $\Pi[---] \ldots[\ldots] \alpha \mathrm{lc}$ eic $\Delta \tilde{\eta} \lambda \mathrm{o}[v$

$\Pi^{28}$ fr. $1, \Pi^{26}$ fr. $14\|\Pi[\alpha \lambda \lambda] \eta\|_{i}[\tau] \alpha$ ic Snell $\left(1964^{3}\right)$ 105, obl. Prodi (2016) 1166 n. 95 :

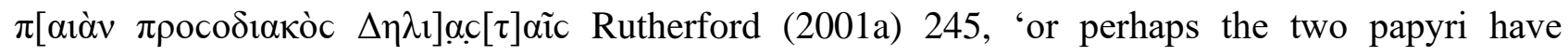
different titles'

68 Pae. 8 = B2 Rutherford : $\Delta \varepsilon \lambda]$

$\Pi^{45}$ fr. 5

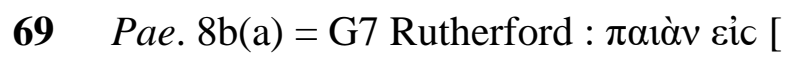

$\Pi^{45}$ fr. 1

70 Pae. $* 19=$ G8.1f. Rutherford : П] $\alpha$ píoıc [

$\Pi^{26}$ fr. 16 || suppl. Maehler $\left(1989^{8}\right) 62$

Prosodia

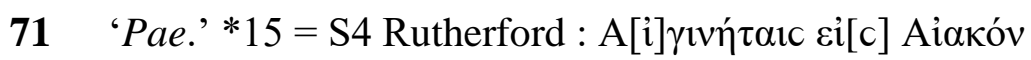

$\Pi^{29}$ fr. 1

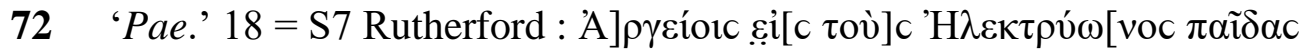




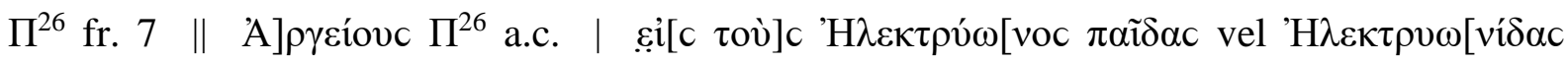
D’Alessio (1997) 41

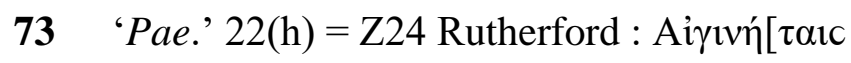

$\Pi^{26}$ fr. 86 || idem ac 64 sec. D’Alessio (1997) 37 adn. 92

74 Z28 Rutherford : $\tau$ oĩc $\alpha$ ขoĩc

$\Pi^{29}$ fr. 3

Dithyrambi

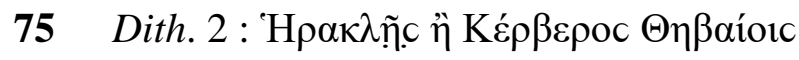

$\Pi^{9}$ fr. 1

Parthenia?

*76 fr. 94b Snell-Maehler : A. $[\gamma \alpha c 1 \kappa \lambda \varepsilon \tilde{\imath}$

$\Pi^{10}$ col. ii $\|$ Prodi (2014) 104 n. 25 post Lehnus (1984) 78

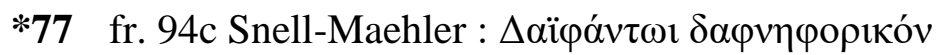

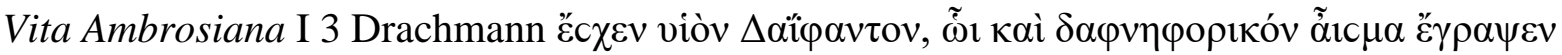

Separata a Partheniis

*78 frr. 95-*100 Snell-Maehler : cic Пóva

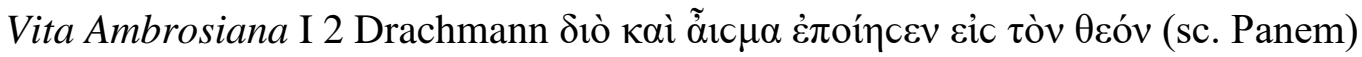

Hyporchemata

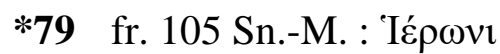

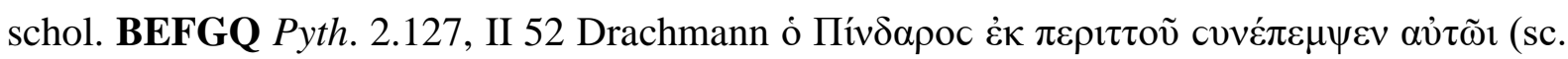

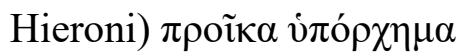

*80 fr. 109-10 Sn.-M. : $\Theta \eta \beta \alpha$ ĩorc 


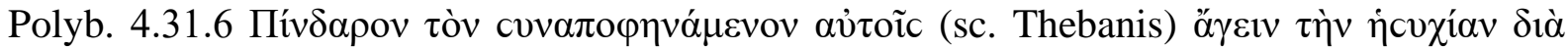
$\tau \tilde{\omega} v \delta \varepsilon \tau \tilde{\omega} \nu \pi 0 i \eta \mu \alpha ́ \tau \omega \nu$

\section{Encomia}

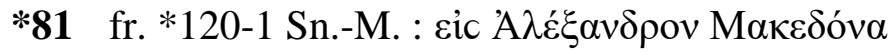

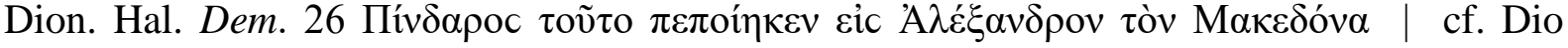
Chrys. Or. 2.33

\section{Threni}

82 fr. 128c Snell-Maehler = 56 Cannatà Fera : ]v

$\Pi^{32}$ fr. 4(b)

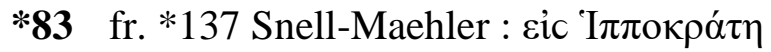

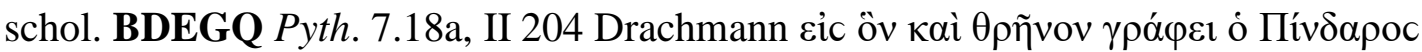

\section{Incerta}

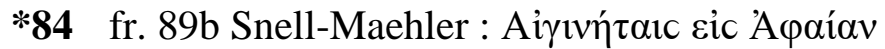

Paus. 2.30.3 Å

85 fr. 140b Snell-Maehler = G9 Rutherford : ]. $\alpha$. ọıc [ - - - ]. . [ - - - ]. . [ - - - Ȧó] $\lambda \lambda(\omega v \alpha)$

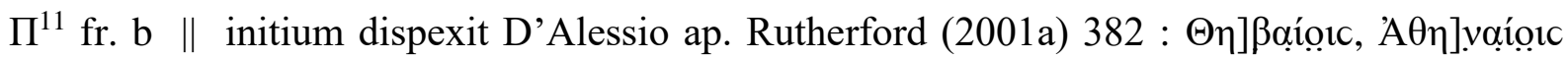

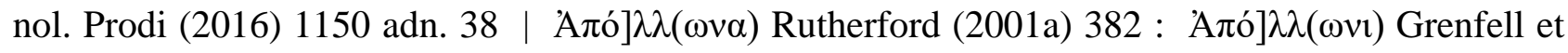
Hunt (1903) 17

86 Z27 Rutherford : $\Lambda \varepsilon[$

$\Pi^{26}$ fr. 94

\section{Bacchylides}




\section{Epinicia}

$87 \quad 2: \tau \tilde{\omega} \iota \alpha \jmath ่ \tau \tilde{\imath} \iota$

\section{$\mathbf{A}^{2}$}

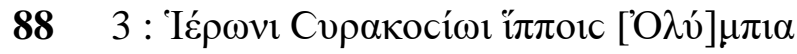

\section{$\mathbf{A}^{3}$}

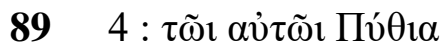

\section{$\mathbf{A}^{3}$}

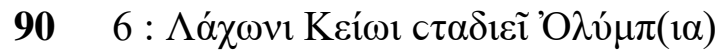
$\mathbf{A}^{3}$

$917: \tau \tilde{\omega} \iota \alpha \hat{\tau} \tau \tilde{\omega} 1$

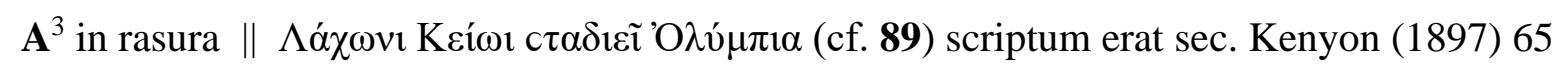

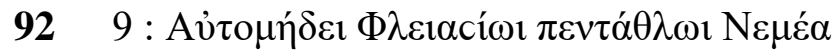

\section{$\mathbf{A}^{3}$}

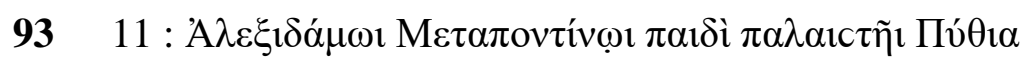
$\mathbf{A}^{3}$

9412 : Tعıcíaı Aỉ

\section{$\mathbf{A}^{3}$}

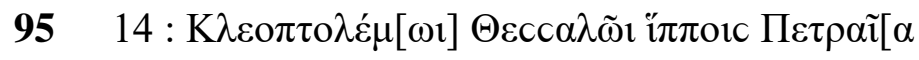

\section{$\mathbf{A}^{3}$}

96 14B : $] \alpha[---] \pi \alpha$

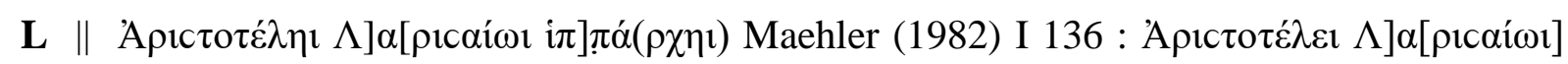

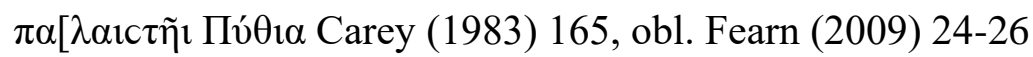

\section{Dithyrambi}

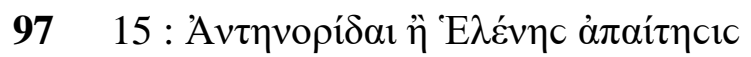

\section{$\mathbf{A}^{3} \mathbf{O}$}




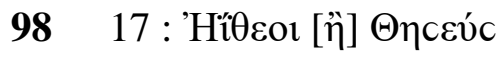

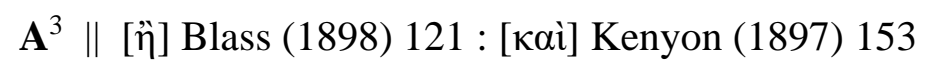

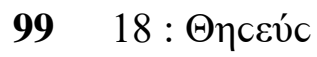

$\mathbf{A}^{3}$

10019 : 'I⿳亠 AA $\theta$ quaíorc

$\mathbf{A}^{2}$

10120 : 'I $\delta \alpha c \Lambda \alpha \kappa \varepsilon \delta \alpha \mu$ ovíorc

$\mathbf{A}^{2}$

$102 * * 23$ (= fr. dub. 60 Maehler?) : Kácc] $\alpha v \delta \rho \alpha$

B $\|$ v. infra, pp. 000

103 fr. dub. 61 Maehler : $\Lambda \varepsilon v \kappa i ́ \pi \pi 1 \delta \varepsilon c$

PSI 1181

Hymni ?

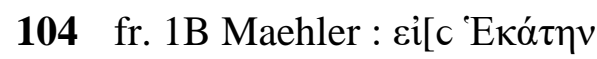

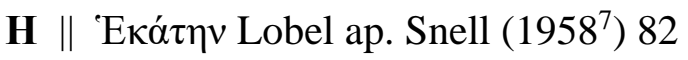

'Encomia'

105 fr. *20B Maehler : ]v [-- - ] [ - - ].

$\left.\mathbf{P}^{2} \| A \lambda \varepsilon \xi \dot{\alpha}\right] v[\delta \rho \omega \imath$ A $\mu \nu ́ v \tau] \alpha$ Grenfell et Hunt (1915) 69, obl. D’Alessio : incertum utrum titulus sit

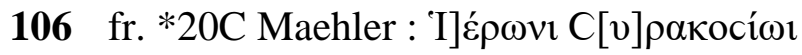

$\mathbf{P}^{2}$

\section{Notes}




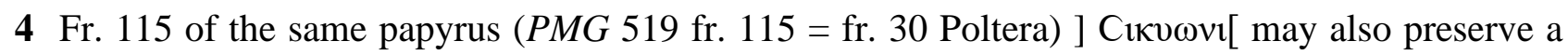
portion of a title, but the supplement is not certain and nothing clearly marks the writing as that of a title. See Poltera (2008) 345.

8 Poltera's radical intervention presupposes (i) a confusion of names (which in and of itself is easy

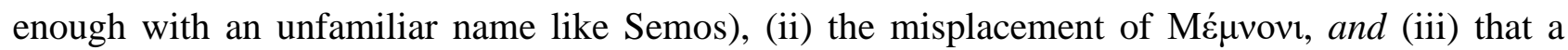
hapless scribe managed to extract $\delta \imath \theta \rho \alpha \dot{\alpha} \mu \beta \omega$ from a mere $\delta$. If we are adamant that Simonides' Dithyrambs did not have a subsection of Deliaka (the likes of 97?) and that the transmitted text is accordingly corrupt, Schneidewin's emendation is more economical. On subsections in Simonides' Epinicians see p. 000 n. 000 above.

*9-*10 The Suda lists a Battle at the Artemision in elegiacs and a Battle at Salamis in lyric metre, but Priscian's quotation of PMG 533a-b shows that the (or at least 'a') Battle at the Artemision was in lyric metre. Bergk (1866-673) III 1145 argued that the Suda had simply inverted the correct metres, but if Parsons (1992) 6, 41 is correct in recognising a description of the battle in P.Oxy. 3965 fr. 20, 'the busy poet celebrated (or mentioned) the same victory twice' (ibid. 6). For a fuller discussion see Kowerski (2005) 4-16 with bibliography. What the metre of the 'battle at Salamis' was remains uncertain: see Rutherford (2001b) 37-38, Obbink (2001) 82.

11 It is unclear whether these Prayers were a poem (and of what sort) or a book. Pontani (2012) suggests that they were a poem, one of the Dithyrambs; Rutherford (forthcoming) inclines towards a separate book.

12 Given the title, one would suppose that the Europa was a dithyramb: so already Bergk (1878$\left.82^{4}\right)$ III 399.

*46 The scholiast's remark that 'some say that both Deinias himself and his father were stadionrunners' suggests that the title had chosen the other possibility, viz. that they won in the diaulos. aủ óv may suggest that only Deinias was named in the title, but it cannot be excluded that also his father Megas was. 
47 It is likely that the brief description of the Hekatombaia in the headnote (BD, III 165 Drachmann) stemmed from a (now lost) reference to that agon in the title, just as $\mathbf{5 8}$ and $\mathbf{5 9}$ probably occasioned the headnotes on $\Pi^{25}$ B frr. 14.i.28, 17.11-18; cf. 15 with inscr. A Ol. 3, I 105 Drachmann. The same could be suggested of Nem. 9, cf. inscr. BD, III 149 Drachmann. See further p. 000 above.

*52 If the title had said that the ode was addressed to Pytheas alone, Kallistratos would not have needed to specify as much. His remark, and the scholiast's decision to quote it, suggest that the title referred to both Pytheas and Phylakidas. For a contrary view see Negri (2004) 197-98. For titles referring to multiple dedicatees cf. $\mathbf{2}, \mathbf{2 0}, * \mathbf{5 3}, \mathbf{5 8}$, and perhaps $* \mathbf{5 4}$.

*62 Despite mentioning different deities as dedicatees of the hymn, the Vita Ambrosiana (followed by Eustathius) and Pausanias must refer to the same composition, as is suggested by their respective quotations from the text. Lehnus (1973b) 5-11 argues persuasively that the Vita is correct and suggests an explanation for Pausanias' mistake; see also Daude et al. (2013) 89 and n. 62.

75 The traces above these words in the papyrus are not a further line of the title, as thought by several editors, but the bottom of an asteriskos marking the end of the previous poem: Prodi (2016) 1164.

82 It seems that (only) this letter, and not (also) the note written in a tiny script between the koronis and the column, belongs to the title: Prodi (2016) 1163. Two alternatives can be conceived - that ]v is the final letter of an extraordinarily long line of the previous column, or an unmarked stichometric letter marking line 1,300 of the book - but neither carries overwhelming conviction.

86 Given the small size of the fragment and the crowd of annotations around the text, it is difficult to determine with certainty that $\lambda \varepsilon[$ is the beginning of a title, but this seems the most satisfactory explanation of the data: Prodi (2016) 1166-1167. How to supplement it remains an open question. 
As can be seen at a glance from the Catalogue, titles come in more or less fixed patterns book by book, that is (at least notionally) genre by genre. This is confirmed by how these patterns tend to be the same in all three authors, unless the different secondary arrangement of one genre into multiple books required otherwise. An example of the latter are the victory odes. Pindar's were divided by games, with few exceptions. ${ }^{38}$ Simonides' were arranged by discipline, although the details are not entirely clear. ${ }^{39}$ Bacchylides had just enough to fill one book, so the problem did not pose itself. ${ }^{40}$ The information offered by their titles differs accordingly. The identification of the victor with name and (usually) ethnic in the dative, as well as the indication $\pi \alpha \_\delta$ when applicable, is common to all three poets. ${ }^{41}$ Simonides' titles also specified the discipline, again in the dative, when a book included several $(\mathbf{2}, \mathbf{4}) .{ }^{42}$ Pindar's did the same, because his book-titles were silent on the subject (13-60); for athletic and musical disciplines the dative is an apposition to the victor's name, while for equestrian disciplines it is instrumental. ${ }^{43}$ Bacchylides' indicated both the discipline and the games, the latter in an accusative that may have been envisaged as depending on an implied

\footnotetext{
${ }^{38}$ The last three Nemeans are not actually Nemean odes (inscr. BD Nem. 9, III 150 Drachmann), and we know from $\Pi^{25}$ that at least two poems at (or near) the end of the Isthmians are not actually Isthmian odes
} $(\mathbf{5 9}, \mathbf{6 0})$. The ancient debate about the proper classification of Pyth. 2 (inscr. DEFGQ, II 31 Drachmann) is of a different order, in that the ode was classed among the Pythians because arguably (if controversially) connected to the Pythian games.

${ }^{39}$ See Lobel (1959b) 89, Obbink (2001) 75-77, Poltera (2008) 12-14.

${ }^{40}$ First noted by Blass (1898) iv.

${ }^{41}$ The specification $\pi \alpha \iota \delta i$ is not actually attested for Simonides; $\pi \alpha \iota c i ́ v$ in $\mathbf{2}$ is a different matter.

${ }^{42}$ See Lobel (1959b) 89. I do not take into account P.Stras. inv. 1406-1409, attributed to Simonides' Epinicians for Runners by Snell (1937) 98 and mentioned in this connexion by B.-J. Schröder (1999) 165: as Ucciardello (2016) demonstrates, the text it preserves is not Simonides but the sayings of Secundus the Silent Philosopher.

${ }^{43}$ B.-J. Schröder (1999) 165. 


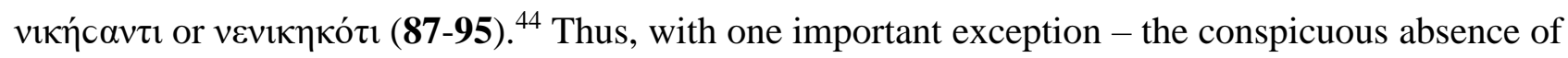
any reference to games in Simonides' surviving titles ${ }^{45}$ - the information given by book-titles and poem-titles combined is much the same in all three authors. It is the difference in the former that necessitates a complementary difference in the latter.

In the case of other books, the picture is one of greater cross-author consistency, scarcer though the evidence is. The titles of both Pindar's and Simonides's Paeans indicated the community that was thought to have commissioned and presumably performed the ode, in the dative, and the sanctuary to which the song was said to be destined, with cic and the accusative (5, *63-64, 66-69). ${ }^{46}$ The reason why the deity was not indicated is probably that paeans were taken to

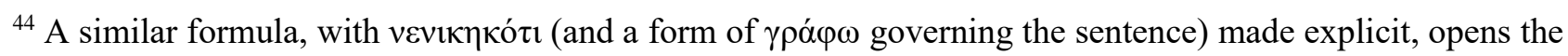
headnotes to several of Pindar's odes, see p. 000 below.

${ }^{45}$ The only title of a Simonidean victory ode where the games were certainly not indicated is $2(P M G 511=$ fr. 7 Poltera). Line 5 of the poem clearly intimates a Pythian victory, but given the state of the text there is a possibility - though I should not say a likelihood - that the $i \pi \pi \circ \delta \rho[o \mu] i \alpha[1$ (?) of the following line refer to a different set of games rather than specify the victory won in the Pythic contest. If the former, then the slot (as it were) for the indication of the games may have been left empty out of uncertainty. But this hypothesis has little to recommend it, unless the mention of Zeus at the very beginning of the ode is taken as 'an oblique reference to an Olympian or Nemean victory' (Lobel [1959b] 89), which it might but need not be. Also, if the peculiar format for the indication of the victors is taken from a victory catalogue (a likelihood on which see pp. 000-000 below), the catalogue would necessarily have clarified the matter - unless, of course, the

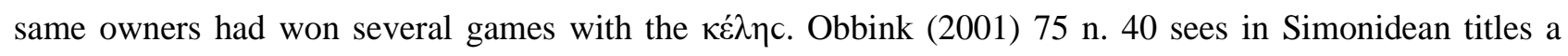
general lack of information about the games and explains it by suggesting 'that Simonides did not regularly mention in his epinicia (as Pindar and Bacchylides did) the site of the competition, and thus his epinicians were, as far as this differentia was concerned, transparent to Hellenistic editors'.

${ }^{46}$ Given the parallel of Callimachus' Hymn to Delos, where the island is clearly construed as the honorand in the very text of the poem, one may wonder whether sic + accusative in our titles was perhaps meant in the usual sense: a paean in honour of Delphi, Delos, etc. But there is no evidence that Paeans so titled were 
be dedicated to Apollo by default. 65 follows the titling pattern of the Prosodia even in its placement among the Paeans, where indeed it is labelled a $\pi \rho \circ\left[[o ́] \delta 1[o] v .{ }^{47}\right.$ Dithyrambs had a title 'proper' - or two - that designated the poem with the name of a central character or (less frequently) event, in the nominative; at least in some cases it was followed by an indication of the commissioning community, in the dative. ${ }^{48}$ Hymns must have been titled with sic and the accusative of the relevant deity, like the Homeric Hymns and those of Callimachus (and many others). It appears from Pausanias' citation of $* \mathbf{6 1}$ (Pindar) that at least some titles of Hymns included the usual dative for the community as well, although $\mathbf{1 0 3}$ (Bacchylides) may be an example of the contrary. ${ }^{49}$ Titles of Threnoi must have indicated the deceased whom the poem mourned, as all three of our sources suggest $(\mathbf{7}, \mathbf{8 2}, * \mathbf{8 3})$. The Pindaric scholiast who cites $* \mathbf{8 3}$ would have had no syntactical reason to turn a dative into the form with eic and the accusative; the latter form is supported by $\mathbf{8 2}$, if the one letter in question is rightly understood as the end of a title (see $n$.).

really taken to be dedicated more to the place than to the god. One may rather suppose that Callimachus riffed on an existing bibliographical practice - which would then have predated him and, consequently, the "standard" Alexandrian Pindar - and composed a poem that turned the topographical sic $\Delta \tilde{\eta} \lambda$ ov of (especially) 67 into the more common hymnic sense, with a very Callimachus-like twist.

${ }^{47}$ See p. 000 and n. 000 below.

${ }^{48}$ For Bacchylides see pp. 000-000 above. Simonides' case is hard to judge: both the relevant titles $(\mathbf{8}, \mathbf{1 1})$ are attested only indirectly, so the absence of a dative indicating the community in the sources could be due to omission on the part of the source. Moreover, neither is above doubt: the passage that preserves $\mathbf{8}$ is probably corrupt, although to an uncertain extent, and Aristophanes of Byzantium's citation of $\mathbf{1 1}$ does not explicitly state that the Europe was one of the Dithyrambs (but see the respective $n n$.).

${ }^{49}$ Note, however, that the attribution of $\mathbf{H}$ to the Hymns is conjectural: Lobel (1956a) 40. All that is preserved of the title is $\varepsilon[$, but the scarcity of city names beginning with that combination of letters supports Lobel's supplement $\varepsilon \hat{i}[\mathrm{c}$. 
The evidence for other books is much thinner and limited to Pindar. Titles of Partheneia - or at least of the Theban $\delta \alpha \varphi v \eta \varphi$ оки́ - probably indicated the daphnephoros, the boy who played a leading role in the ceremony and was therefore construed as the dedicatee of the ode $(* 76-* 77)$. The one title that is known (indirectly) from the $\kappa \varepsilon \chi \omega \rho \imath \mu \mu \varepsilon v \alpha \tau \tilde{\omega} \nu \pi \alpha \rho \theta \varepsilon v \varepsilon i ́ \omega v(* 78)$ follows the same format as the Hymns, but too little is known about this strangely-titled book to assume consistency of titles, or indeed of generic identification, within it. Titles of Hyporchemes may have referred to the individual or community that commissioned the ode $(* \mathbf{7 9}, * \mathbf{8 0})$, although it is not certain that the two sources derive the respective information from the titles. In both passages the dative is required by the syntax and thus cannot be assumed to be a literal quotation. On the other hand, one wonders what other form that indication could have taken: one does not normally find a community introduced by eic in such context. There is a fair amount of first-hand evidence for titles of Prosodia, which are now one of the best-attested parts of Pindar's auvre at least in terms of the number of manuscripts. ${ }^{50}$ The indication of the commissioning community in the dative is followed by that of the deity or hero to whom the song is dedicated $(\mathbf{6 5}, \mathbf{7 1 - 7 3})$, the same format as the Hymns may have had. The incerta $* \mathbf{8 4}$ and $\mathbf{8 5}$, which also follow that format, may therefore have been part of either book. ${ }^{51}$

One case remains to be discussed: the books of Pindar and Bacchylides commonly known as Enkomia. That this was the title in Pindar's case is guaranteed by the Vita Ambrosiana (I 3 Drachmann) and by the anonymous biography preserved by P.Oxy. 2438 (col. ii.38). The latter goes on to suggest that the book contained a sub-group of poems with a distinct identity, perhaps cкó $\lambda ı \alpha$

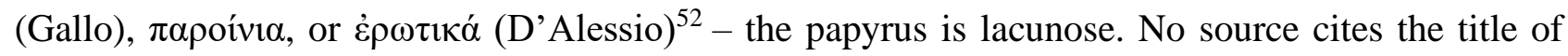

\footnotetext{
${ }^{50}$ On identifying papyri of the Prosodia $\left(\Pi^{7}, \Pi^{8}, \Pi^{29}\right.$, and parts of $\left.\Pi^{26}\right)$ see the seminal article by D'Alessio
} (1997). I ventured some additional speculation on $\Pi^{34}$ in Prodi (2013) 53-55. The Prosodia are also one of three plausible candidates for the attribution of $\Pi^{11}$.

${ }^{51}$ On 85 see also Prodi (2016) 1149-1150.

${ }^{52}$ Gallo (1968) 30, D’Alessio (2000), (2016) 83 n. 77. 
Bacchylides' equivalent, fragments of which are preserved by $\mathbf{P}$ and $\mathbf{Q}+\mathbf{U} .^{53}$ Athenaeus $15.667 \mathrm{c}$

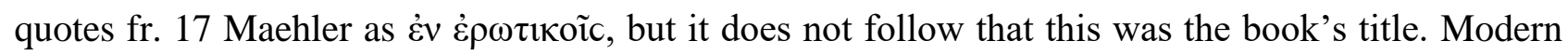
editors generally call it Enkomia, like Pindar's book, but Giambattista D’Alessio argues that Skolia or Paroinia is a likelier alternative. ${ }^{54}$ Another possibility, suggested by David Fearn on the basis of the Pindaric parallel, is that Skolia or Paroinia was the title of a subsection within a book of Enkomia. ${ }^{55}$ Whatever the case, we know from 105 that (some) titles in the book indicated the person for whom the poem had been composed, in the dative. ${ }^{56}$ Perhaps because of the envisaged similarity with the better-known Epinicians, datives of the same sort had long been conjectured as the titles of Pindar's Enkomia, none of which is directly attested. However, our source for $* \mathbf{8 1}$ presents the honorand's name not in the dative but in the accusative with eic, despite having no obvious syntactic obligation to do so. The presence of datives in the titles of Pindar's Enkomia is thus open to question. If there was indeed a sub-group of Skolia (or Paroinia) within Pindar's book of Enkomia, and if Bacchylides' corresponding book was titled Skolia (or Paroinia) or included a distinct sub-section of such poems, it is also possible that both forms - the plain dative and Eic with the accusative - were in use: poems regarded as skolia may have been titled with a dative indicating the person for whose drinking-party and at whose behest the song had been composed, while for

\footnotetext{
${ }^{53}$ On $\mathbf{Q}$ and $\mathbf{U}$ as fragments of one manuscript see Irigoin (1993) xxxvii, D’Alessio (2016) 79.

${ }^{54}$ D'Alessio (2016) 82-83.

${ }^{55}$ Fearn (2007) 27-28 n. 2.

${ }^{56}$ One important assumption must be flagged here: namely, that titles on $\mathbf{U}$, although written long after the text was copied, were nevertheless copied from an exemplar rather than conjectured on the basis of the text itself. If the latter was the case, then they no longer testify reliably to the format that was used in the Alexandrian Bacchylides (although the person responsible may have been aware of the format even if he was not copying them directly). The absence of clear comparanda for such delayed suppletion of titles makes the case hard to adjudicate. For a partly similar case involving Bacchylides' A (but without the large time lag) see pp. 000-000 above.
} 
poems regarded as enkomia proper - poems concerned more directly with praise of the individual in question - the phrase with sic and the accusative was felt to be more appropriate. This, however, would create a strange disparity with the Epinicians, which are surely no less encomiastic in nature.

\section{Functions and implications}

Generally speaking, titles can serve two broad kinds of purposes, one largely text-internal and one largely text-external: orientation, whereby the title guides the reader's expectations about, and understanding of, the text; and reference, whereby the text can intelligibly be cited outside, and to some extent independently of, itself by means of its title. ${ }^{57}$ In our case, the referential function is less prominent than we might expect from our experience as twenty-first-century readers of mostly recent (or recently-processed) material, or even from our knowledge of other areas of the classics. Ancient authors cited lyric far more frequently by book than they did by any smaller unit. Even when they did refer to a specific composition within a book, its title was not the default mode of reference. Another was the incipit. Its use of which was inevitable when the text in question had no individual identifier outside of itself (or when it was necessary to link securely such an identifier to

\footnotetext{
${ }^{57}$ Levenston (1978) 63 makes much the same distinction, using the terms 'contextualization' and 'reference'. “"Contextualising a poem" means establishing the participants, the immediate situation, the wider situation, the thesis and other components of the context of situation that determine the instantial meaning of the poem, considered as utterance. And one or more of these components, in greater or lesser detail, can be named and described in the title' (ibid.). Following B.-J. Schröder (1999) 164, I prefer the term 'orientation' so as better to bring out the implicitly authoritative status of titles vis-à-vis the reader, on which see Hoek (1981) 2 and especially Ferry (1996) 2. Cf. also Genette (1987) 76-78 on the separation between the referential use of a title ('strict designation, or identification', 77) and the motivation for the choice of that title.
} 
the text itself), but it could be used even when the poem had a title of its own. ${ }^{58}$ Our now preferred mode of citation, the serial number of the poem within the book, was used exceedingly rarely. To my knowledge, its only sustained use in Greek-speaking antiquity is in the metrical scholia to Pindar, and perhaps in one fragmentary work of scholarship on Alcaeus (P.Oxy. $2734=C L G P$ Alcaeus 15). ${ }^{59}$ Otherwise it seems to be restricted to the first ode in a book, perhaps on account of its special position or its particular importance within the book or the author's work. ${ }^{60}$ This should come as no surprise: in none of our authors' surviving papyri are the individual odes numbered.

It would be idle to speculate on the reasons for the simultaneous presence of these competing uses, for the failure of any of them to prevail, or indeed for the enduring preference for citation by book over citation by individual poem. However, this situation highlights one related fact: namely, that titles were not meant to be unique identifiers of the respective poems. Even in the titles of

\footnotetext{
${ }^{58}$ See Nachmanson (1969) 37-45, Holtz (1997) 474-77, Borgo (2007). For Pindaric examples cf. e.g. Ol. 6 in
} P.Oxy. 2438 col. ii.17-18; fr. 29 Snell-Maehler in schol. BD Nem. 10.1a, III 165 Drachmann; Isthm. 5, 6 and Nem. 5 in inscrr. a-b BD Isthm. 5, III 241 Drachmann.

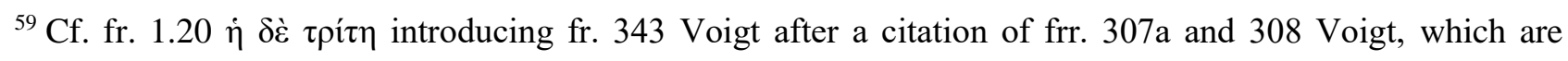
otherwise known to have been the first and second poems in Alcaeus' first book. The exact nature of P.Oxy. 2734 is disputed: see Lobel (1968) 2 ('a set of summaries of the contents of each poem'), Porro (1994) 13148 and in CLGP ad loc.

${ }^{60}$ The only certain example in our three poets is Pind. fr. 29 Snell-Maehler, cited by Lucian. 58.19 and

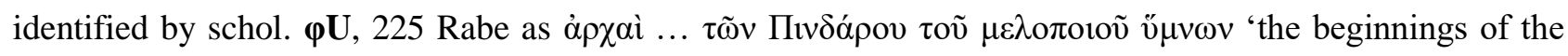
Hymns of the melic poet Pindar'. Lehnus (1973a) 398 and Lavecchia (2000) 274-76 suggest that $\dot{\varepsilon} v \delta \dot{\varepsilon} \tau \tilde{\omega} 1$ $\pi \rho \omega ́ \tau \omega \iota \tau \tilde{\omega} \nu \delta 1 \theta v \rho \alpha ́ \mu \beta \omega v$ 'in the first of the Dithyrambs' in schol. BCEQ Ol. 13.25c, I 361 Drachmann, $\delta 1 \theta v \rho \alpha ́ \mu \beta \omega v \pi \rho \omega ́ \tau \omega \iota$ in Etym. Magn. 1317 Gaisford, and $\dot{\varepsilon} v \tau \tilde{\omega} \imath \pi \rho \omega ́ \tau \omega \iota ~ \tau \tilde{\omega} v \pi \alpha \rho \theta \varepsilon v \varepsilon i ́ \omega v$ 'in the first of the Partheneia' in schol. REГLh Ar. Ach. 720 Wilson (Pind. frr. 71, 72, and 94d Snell-Maehler respectively) indicate the first poem of the book, not the first book of the work, but this hypothesis runs against the established meaning of such phrases; see Filoni (2007) 75-76 and already Turyn (1948) 290. 
Dithyrambs - those titles that, as we have seen, approximate our modern usage the most duplication can be found, and at very close quarters too. O१cev́c is the title of two consecutive Dithyrambs of Bacchylides, 17 and 18 (98-99). In the second of these it is even an alternative title introduced by $\dddot{\eta}:$ a far cry from any disambiguating function. ${ }^{61}$ Other books fare no differently. Far from attempting to distinguish accurately between poems by means of their titles, the editor habitually flagged the notional reoccurrence of a title with substitutory phrases such as $\tau \tilde{\omega} 1 \alpha \cup j \tau \tilde{\omega}$,

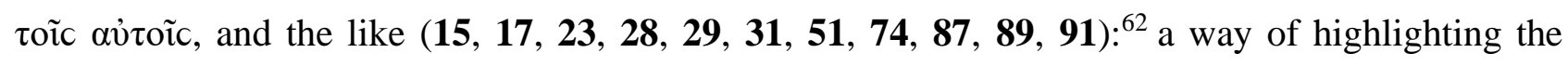
repetition while ostensibly avoiding it, essentially reducing the title to a mere cross-reference.

A more important and complex role that a title fulfils is to orient the reader's understanding of the piece in question. Much as labels in a museum tell the viewer what type of object they are seeing, its function, its provenance, a rough or exact date, and perhaps its individual name, the titles of our poems filled in the information that the reader was thought to need or have use for. This applied especially to context, loosely intended, which otherwise for the reader was altogether lost. This dithyramb was written for the Athenians; this epinician, for Hieron of Syracuse; this hymn, to Poseidon. This information may well have been actually extracted from the text itself by whoever etablished the title, but in the original production of the song it would have been independent of it and situated in the reality outside it. The details of this Orientierungshilfe (Schröder's term) ${ }^{63}$ with regard to the editor's purposes and his readers' responses are beyond recovery, but one can fruitfully speculate on the effects that titles may have had and the assumptions that arguably underlay them.

The way in which a title presents extra-textual information upfront, before and outside the poem, contributes to detaching the text from its mere textuality by enriching it with contextual, or

\footnotetext{
${ }^{61}$ On alternative titles see also pp. 000-000 below.

${ }^{62}$ See B.-J. Schröder (1999) 165, Prodi (2013) 57.

${ }^{63}$ B.-J. Schröder (1999) 164.
} 
purportedly contextual, elements. It conjures up an extra-textual "broader picture" to which the text can be anchored, effectively fictionalising it at the same time as ostensibly framing it in reality. Thus titles fulfil a performative function of sorts: by asserting that a certain poem was composed for the Aeginetans or dedicated to Pan, in the eyes of the obedient reader the title makes it - in a way -

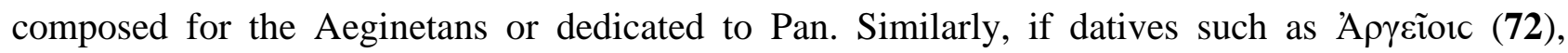

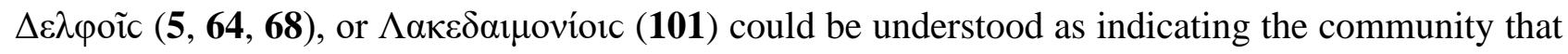

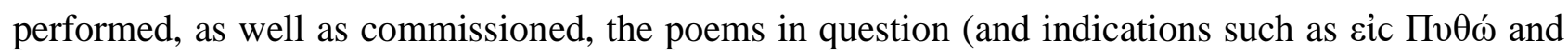
Eic $\Delta \tilde{\eta} \lambda$ ov do imply a modicum of interest in the performance of the odes), then these portions of the relevant titles may have played a quasi-dramatic part, functioning as a sort of speakers' tags for the text itself. From the opposite viewpoint, titles also belie these extra-textual endeavours through their very textuality: by inscribing originally non-written, non-textual elements in written form they seal the textualisation that these compositions had undergone before reaching their resting place in the Library.

The role of titles in the librification of our authors - in the process of integrating their poems into books, that is, of turning poems from self-standing pieces into parts of a larger entity - invites further comment. There is no hard evidence that these two processes, the coalescence of poems into books and the definitive attribution of titles to them, were simultaneous or interrelated. All we know is that both were probably finalised in the "standard" editions of the respective authors, and that both probably had an indeterminable number of precedents about which, in turn, very little is known. Nevertheless, titles have an important role in playing out the poems' belonging in the book in which they are situated. With their standardised patterns, titles highlight each poem's pertinence to the book by subsuming the difference that each title enunciates into the larger unity that their uniformity underscores.

This is all the more significant in view of the outwardly close association between books and genres in our authors. For all the complications, theoretical and practical, of ancient conceptualisations of the various genres of lyric poetry, nevertheless the book-titles of Simonides, 
Pindar, and Bacchylides simply advertise the respective books as a plurality of entities identifiable as poems of a specific kind: ${ }^{64}$ the Paeans are a collection of paeans, the Hymns one of hymns, and so forth. Indeed, one effect of such ostensibly transparent book-titles is to conceal the book - and its fundamental constructedness as such - behind the poems it brings together, grouped and labelled according to the genre to which they are said to belong. From the generic perspective, as a result, poem-titles simultaneously individualise each poem through the specific indications that they offer, and contribute to characterising the genre through the elements that they consistently put forward for that purpose. This format also enables the editor to flag pieces regarded as extravagant by means of a simple deviation from the general pattern, such as the generic subtitles appended to 59, $62, \mathbf{6 4}$, and 65. It is a pity that the titles of Nemeans 9 and 11 and the relevant portion of $\mathbf{4 7}$ are lost (but see n.), because they might have provided an interesting term of comparison; see also $\mathbf{9 5 .}$

The titles of individual poems are necessarily in a dialogue with the title of the book. ${ }^{65}$ The reader is required to combine the information provided on both levels in order to read a dithyramb for the Thebans called Heracles or Cerberus or an epinician for Hagesias of Syracuse who won the Olympics with the mule cart $(\mathbf{7 4}, \mathbf{1 8})$. The addition of a generic subtitle complicates this dialogue.

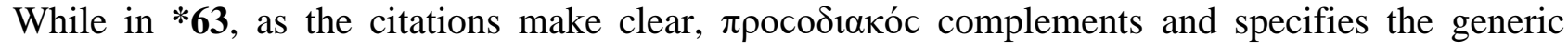

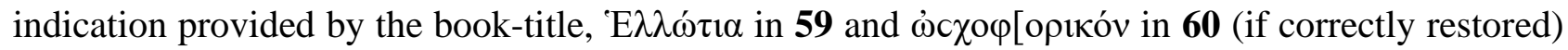
appear rather to overrule it: a 'prosodiac paean' is still a paean, but an oschophoric is not an Isthmian ode. ${ }^{66}$ Conversely, the indication of further games attached to $\mathbf{2 4 ,} \mathbf{3 3}$, and perhaps $* \mathbf{5 0}$ and *53 (if genuine) clearly constitute additions to, not a denial of, the information offered by the booktitle. Given the particularly complex status of 'Paean' $6(\mathrm{~b}),{ }^{67}$ the only piece by Pindar that is known

\footnotetext{
${ }^{64}$ Cf. B.-J. Schröder (1999) 63-64.
}

${ }^{65}$ Compare the remarks on the cooperation of book-titles and poem-titles in Levenston (1978) 69-70.

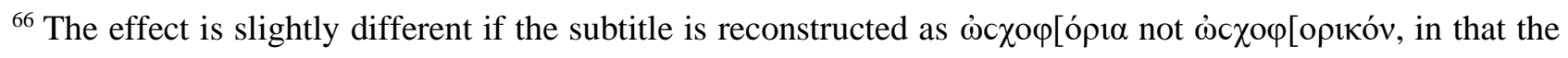
correction would be phrased indirectly in terms of the contest won, not directly in generic terms.

${ }^{67}$ See n. 000 above. 
to have appeared in two different places in his Alexandrian corpus, one does not quite know what to make of the subtitle $\pi \rho \operatorname{coc}^{\delta}$ เov (65). Does it imply that (genre-wise) the piece, being a prosodion, is not a paean? Or does it simply state that (book-wise) the poem was a Prosodion as well as a Paean? The former is suggested by the twin considerations that the format of the title is the one found in the Prosodia, not the Paeans, and that the editor opted for $\pi \rho$ ocó $\delta 10 v$ over the attested alternative

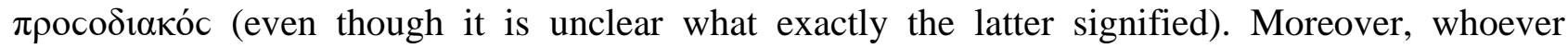

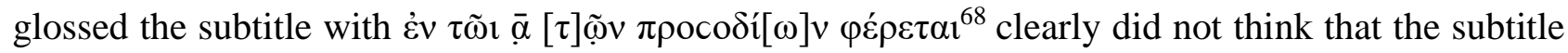
itself was a sufficiently explicit indication that the piece also belonged to the book of the Prosodia. However, the piece was included in both books - unlike frr. 6a.(i)-(1) and 6c Snell-Maehler, which had no book where they should rightfully be - and quite openly presents itself as a paean (127, cf. 182). Like many other aspects of that piece, the subtitle remains mysterious.

The editorial choices that shape the format of titles in each book inconspicuously orient the reader's expectation (ex ante) and understanding (ex post) of what kind of thing the poems - and, by extension, the genre - are. Naturally, a Hymn or a Prosodion are composed in honour of a certain divine or heroic being; but at least a Prosodion (the state of things with the Hymns is less clear, as we saw) is also the expression of a certain community, for whose worship it was composed. Consistently with some ancient conceptualisations of the genre, a Dithyramb is, first and foremost, a story: ${ }^{69}$ whether the poem is named after an individual, or a group, or a specific episode, the title directs the reader's expectations towards a narrative or, more broadly, a presentation of events. (The similarity of dithyrambic titles 'proper' to the titles of other literary forms concerned with the narrative or non-narrative representation of events, such as tragedies or citharodic nomoi, is

\footnotetext{
68 'It is transmitted in the first (book) of the Prosodia' (schol. Pae. 6.124, 604 Rutherford).

${ }^{69}$ Pl. Resp. 3.394b-c, [Plut.] De mus. 1134e (going back to Glaukos of Rhegion?), and by implication the commentary to Bacchyl. **23 (B) quoted below, pp. 000. See Ieranò (1997) 322-5, S. Schröder (1999) 121, D’Alessio (2008), (2013) 119-21.
} 
hardly coincidental.) While a Prosodion and, at least in some cases, a Dithyramb are composed for an entire community, an Epinician or an Enkomion is composed for a single individual. Any factors that might - and in all likelihood did - concretely complicate this neat outline are overlooked or excised. Any public relevance of a privately-commissioned victory ode for a tyrant or prominent citizen, or any less-than-communal concern of a public but privately-sponsored composition such as a dithyramb (but the phenomenon must have been much more widespread) ${ }^{70}$ - all are carefully wrapped away from view, and so inconspicuously that we still find ourselves struggling with their implications for our understanding of the poetry's historical reality.

Another effect worth highlighting is how the datives employed in this connexion foreground, however obliquely, the act of composition. A prosodion for the Argives or a Pythian ode for Telesikrates of Cyrene are a prosodion composed for the Argives and a Pythian ode composed for Telesikrates of Cyrene. In Greek even more than in English, this need for tacit and perhaps unconscious supplementation is almost intrinsic in the syntax. In fact, the scholia routinely make the verbal element explicit: the headnotes to most of Pindar's Epinicians open with statements such

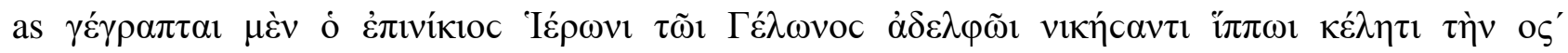

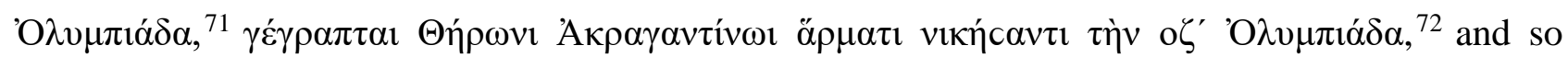
forth, where the choice of $\gamma \rho \alpha \dot{\varphi} \varphi \omega$ as the default verb and of the perfect as the default tense filters the past of the occasion through the permanent present of the written text. As a result, the author and the circumstances of the poem's composition are implicitly brought to the fore. Indeed, titles such

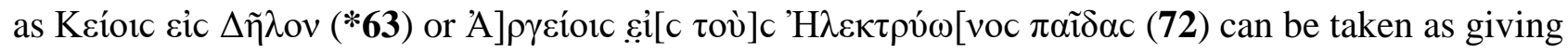

\footnotetext{
${ }^{70}$ See for instance Kurke (2007) on aristocratic display in the Theban Daphnephoria and Fearn (2011a) on possible oligarchic patronage over Aeginetan cult song.

71 'The victory ode is written for Hieron, the brother of Gelon, who won the seventy-sixth Olympiad [476 BC] with the single horse' (inscr. a ADEFHKQ Ol. 1, I 15 Drachmann).

72 'It is written for Theron of Akragas, who won the seventy-seventh Olympiad [actually the seventy-sixth in 476 BC, cf. P.Oxy. 222 col. i.18] with the chariot' (inscr. ABCD ${ }^{\mathbf{a b}} \mathbf{E H Q}$ Ol. 2, I 58 Drachmann).
} 
the essentials of the commission that originated the ode. As they entice the reader to look back in time to that foundational creative moment, these titles not only contextualise the poem, but tell how it came into being.

\section{Evidence}

The factual-sounding snippets of information offered by a title can play a key role in our understanding of the context or destination of a given poem, especially - as is the case for a large part of our sample - when the poem is fragmentary. Therefore, the veracity or otherwise of that information can be a crucial factor for the correctness or otherwise of our reading and interpretation of that poem. Knowing as we do that titles were given their definitive form sometime between the late third and the early second century, to accept unquestioningly whatever a title claims is surely a risky business; but equally unwise would it be to ignore titles altogether out of a vague suspicion of spuriousness without further investigation.

In concrete terms, assessing the veracity of the information provided by titles means examining the material on which the respective editors based their titulatory choices. Such material can be divided into three broad categories: (i) the text itself, (ii) material external to the text but transmitted together with it, and (iii) material independent of the text. The first is the most obvious. Occasional praise poetry like victory odes naturally gives ample information on the addressee of such praise and on the occasion for it. Cult songs will typically make it clear which divinity or hero is being worshipped. Often the setting of such a piece of poetry will also be easy to infer from its text. Examples could be multiplied. The second category, on the other hand, is much shadier. There is no undisputable evidence for its existence, except a reasonable doubt that a few hundred poems can all have reached Alexandria as bare texts with no further information attached to them whatsoever. Simply put, we know nothing precise about how Greek lyric ended up with the scholars of the Museum: while we do know of some who read it and used it, so that we can profitably 
investigate (say) Aristophanes' Simonides or the lyricists in the Peripatos, ${ }^{73}$ the concrete details of its presumably multifarious transmission - how exactly each piece got into the Library, from where, through whose hands, and in what state - are obscure. Finally, the third category represents a variety of potential pieces of evidence, ranging from occasional references by other authors (such as Chamaeleon's account of the commission and performance of fr. ${ }^{*} 122$ Snell-Maehler $)^{74}$ to material that does not refer directly to the texts, such as victory records. Given the considerable uncertainty that surrounds the details, and sometimes the very existence, of extra-textual evidence, it is hard to assess how much in a title derives from the texts itself. On the one hand, our defective knowledge of subsidiary materials often makes it impossible to ascertain whether a certain datum in a title, which may seem to have been derived from the text, was in fact supported by external evidence too, and of what kind. On the other hand, the fragmentary state of so many poems often does not even allow one to see whether the information found in a title could have been inferred from the text. These difficulties, and the conjectural nature that much of the discussion is therefore bound to have, should be kept in mind throughout this final section.

\section{Epinicians}

A convenient starting point, given the uncommonly firm evidence at our disposal, are the Olympic and Pythic victor-lists. The only register of Pythic victors known to us was compiled by

\footnotetext{
${ }^{73}$ Reference is to Rawles (2013) and Hadjimichael, this volume pp. 000-000. Hadjimichael rightly stresses the importance of textuality and of its practicalities, such as the availability of written texts and the uncertainties of their supply from abroad, in fourth-century receptions of the lyricists.

${ }^{74}$ Fr. 35 Martano = 31 Wehrli ap. Ath. 13.573e-f. What Chamaeleon provides is probably for the most part his own reconstruction based on elements he more or less imaginatively extracted from the text itself, see Budin (2008) 150-52: a reminder that information provided by third parties is not necessarily independent, let alone reliable. See also Hadjimichael, this volume p. 000.
} 
Aristotle with his nephew Callisthenes, as we know from a contemporary honorific decree (FD III/1 $400=C I D$ IV 10). ${ }^{75}$ Some fragments are preserved by later sources, which ascribe the work to Aristotle alone (frr. 615-17 Rose, tit. 131 Gigon) if they mention an author at all. ${ }^{76}$ Authoritative victor-lists for the Olympics, apparently associated with a précis of the origins and history of the games, went under the names of Hippias (BNJ 6 F 2) and, once again, Aristotle (tit. 130 Gigon, not in Rose).$^{77}$ Parts of one such list are preserved by P.Oxy. $222+2381$ (BNJ 415). ${ }^{78}$ Other victor-lists included accounts of notable events that occurred in each Olympiad, such as Phlegon of Tralles' 'O $\lambda v \mu \pi i \alpha ́ \delta \varepsilon c(B N J 257 \mathrm{~F} 1-33)$ and the anonymous work preserved by P.Oxy. 2082 (BNJ 257a). ${ }^{79}$ It is unclear if the 'O $\lambda v \mu \pi$ เovĩ $\alpha$ compiled by Eratosthenes (BNJ $241 \mathrm{~F}$ 4-8 and probably 11a-b, 14$15 \mathrm{~b}, 44)$ included a wider spectrum of historical information beyond the history of the games and of athletics more generally; ${ }^{80}$ if they did, P.Oxy. 2802 may plausibly represent a copy of that work. ${ }^{81}$ The scholia to Pindar certainly made use of both Olympic and Pythic victor-lists, which allowed

\footnotetext{
${ }^{75}$ See Bourguet in FD ad loc. and Christesen (2007) 181-86.
}

${ }^{76}$ See the discussion in Rose (1863) 545-50. On the Pythic victor-list see also Spoerri (1988) and Christesen (2007) 179-202, 374-81, with the respective bibliographies.

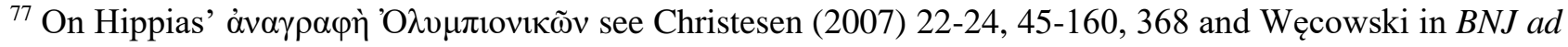
$l o c$. , with the respective bibliographies.

${ }^{78}$ Published by Grenfell and Hunt (1899), see also Christesen (2007) 202-6, 382-84, 519-23 and Anderson in BNJ ad loc.

${ }^{79}$ On Phlegon see Christesen (2007) 32-33, 326-34, 437-44; on P.Oxy. 2082 see Hunt (1927), Christesen (2007) 335-36, 445-47, and Rzepk in BNJ ad loc. Hunt (1927) 82-84 suggested identifying the papyrus with Phlegon's work despite recognising that the level of detail in the narrative was scarcely compatible with how Phlegon must have compressed the relevant period; his suggestion was refuted by De Sanctis (1928) 70-74.

${ }^{80}$ On Eratosthenes' Olympic victor-list see Geus (2002) 323-32 and Christesen (2007) 173-79, 371-73.

${ }^{81}$ De Sanctis (1928) 68-77. 
them to date the victory to which each Olympian and Pythian referred and to provide information on any other victories of the respective honorands. ${ }^{82}$

There is good evidence that titles did the same. At least seven of Pindar's odes are denoted by their title as having been composed for a boy victor, $\pi \alpha 1 \delta i ́(\mathbf{2 0}, \mathbf{2 2}, \mathbf{2 6}, \mathbf{3 6}, \mathbf{3 7}, \mathbf{4 4}, \mathbf{4 5})$. While often the text itself makes clear that the honorand won in the boys' class, in at least two cases it does not: there is no reference to the victor's age in either Olympian 14 or Pythian 11. The information must come from elsewhere. A victor-list, which necessarily reported such information, is the most intuitive solution. ${ }^{83}$ More strikingly, Olympian 14 makes no mention of the contest won, ${ }^{84}$ which, however, is duly noted in the title (26). Similarly, in Olympian 12 Pindar praises Ergoteles' prowess

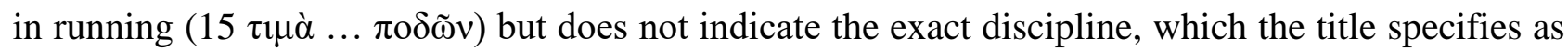
the dolichos (24). ${ }^{85}$ The same argument can be made for Olympian 4, where the contest is never named explicitly (the only reference is 11 óxź $\omega \mathrm{v}$, which can plausibly refer to the apene as well as the four-horse chariot) but the title nonetheless identifies it as the chariot race (16), albeit perhaps

\footnotetext{
${ }^{82}$ The Pythic victor-list used by the Pindar scholia is the Aristotelian work: Christesen (2007) 191, 197-98. Which Olympic list was used is more doubtful. Hippias', Aristotle's, and Eratosthenes' are all plausible candidates. Either of the first two may have been more authoritative on account of its age; the third may have been favoured as a recent, Alexandrian product. Also, like the Pindar scholia themselves, Eratosthenes'

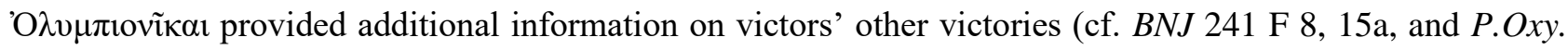
2082 fr. 4). Of course there is no need to assume that only one list was used, or that Eratosthenes' list was independent of Aristotle's for the period that was covered by both (cf. BNJ $241 \mathrm{~F} 7$ ).

${ }^{83}$ Finglass (2007) 10 remarks that the scholiast to Pyth. 11 must have referred to the victor-list in order to identify the relevant victory. The point equally applies to whoever established the title.

${ }^{84}$ Race (1997) I 203.

${ }^{85}$ Confirmed by P.Oxy. 222 col. i.22, cf. Paus. 6.4.11.
} 
mistakenly. ${ }^{86}$ The most economical explanation for the presence of all this extra-textual information is that it comes from the victor-lists.

This is not to say that they were always followed uncritically. If we can trust a puzzling piece of information given by the headnote to Pythian 10 (BDEGQ, II 242 Drachmann), Hippokleas won in the same day both the diaulos and the stadion, but the title follows Pindar's own indication and

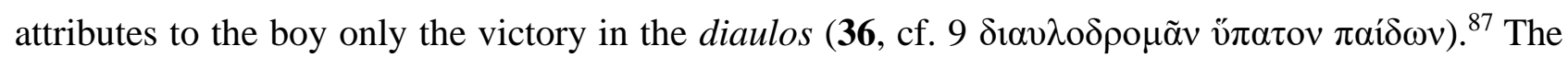
examples of Olympian 4 and, on a different level, Pythian 3 also caution us from assuming that evidence from the victor lists was always used correctly. Nonetheless, there is compelling evidence that the Olympic and Pythic victor lists were consulted as the titles of Pindar's Olympians and Pythians were being established.

There is no such smoking gun for either Bacchylides or Simonides. The title of Bacchylides 6 (90) does not include the specification $\pi \alpha 1 \delta$, which is not obvious from the text but is guaranteed

\footnotetext{
${ }^{86}$ That Psaumis of Camarina won the Olympic chariot race is confirmed by P.Oxy. 222 col. ii.22 (misspelt

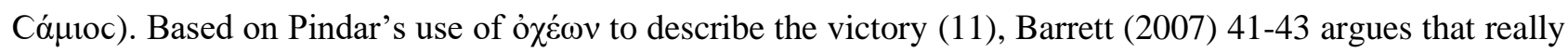
the ode was composed not for that chariot victory but for the one with the mule cart ( $\dot{\alpha} \pi \eta \dot{\eta} \eta$ ) which $\mathrm{Ol} .5$ also, and more explicitly, celebrates. If this is true, the editor's reliance on the victor-list is thrown into even

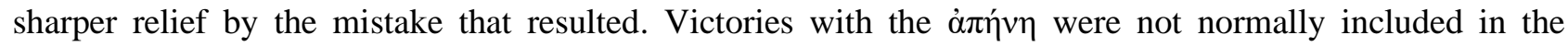
Olympic victor-lists, as can be inferred from inscr. a A Ol. 6, I 153 Drachmann and verified on P.Oxy. 222; consequently, any reliance of $\mathbf{1 7}$ or $\mathbf{1 8}$ on a victor-list can safely be excluded.

${ }^{87}$ That Pindar may have elided entirely a second victory gained in the same games defies belief; the scholiast's conjecture that he had only been paid to celebrate one victory clearly will not do. Bernardini in Gentili et al. $\left(2012^{5}\right) 263$ denies plausibility to the notice of the victory in the stadion altogether, but information such as this does not sound like something one would simply make up. If Hippokleas did only win in the diaulos, then the error may have been Aristotle's or, perhaps more probably, an accidental duplication that intervened at some point of the transmission of the Пvө1ovĩ $\alpha$ before the scholiast made use of them.
} 
by P.Oxy. 222 col. ii.18. This may suggest that the Olympic victor list was not consulted, but an accidental omission in transmission is also a possible explanation. (The presence of $\pi \alpha 1 \delta$ in $\mathbf{9 3}$ is indecisive, as it could have been inferred from the text.) And what was the deal with Simonides' epinician 'for the sons of Aiatios' (2)? Pausanias testifies that a victorious mare could be designated with the name of the father of the present owners in the Olympic victor list he consulted (6.13.10, the sons of Pheidolas). ${ }^{88}$ So any victor list for the competition which our anonymous siblings won

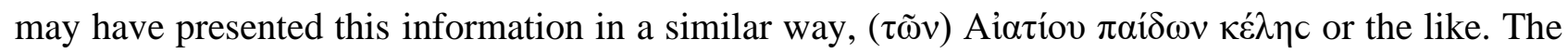
same information might perhaps have been extracted from the text itself, but this would require the assumption that either the poem did not name the $\pi \alpha \tilde{i} \delta \varepsilon c$, or that whoever assigned titles to Simonides' epinicians thought it fit to label them in this way nonetheless.

Things are even more nebulous when we come to Isthmian and Nemean victories. With one exception, which we shall discuss shortly, the scholia to Pindar's Nemeans and Isthmians give no dates for the respective odes. This creates a presupposition that numbered victor-lists (the adjective is important here) were not consistently available. ${ }^{89}$ However, Pausanias states that, at the time of

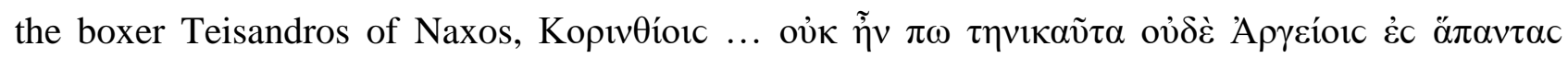

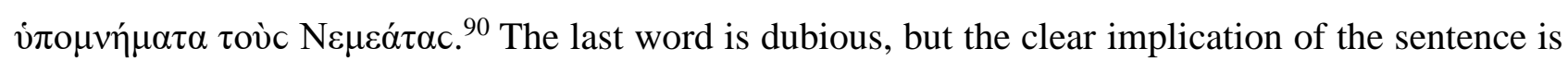

\footnotetext{
${ }^{88}$ See already Lobel (1959b) 89. For a victor-list allegedly registering the joint ownership of a team see also schol. BDEQ Ol. 2.87b, I 82 Drachmann, although in that case the two owners (Theron and Xenokrates of Akragas) seem to have been mentioned individually. However, the notice itself is open to doubt in view of

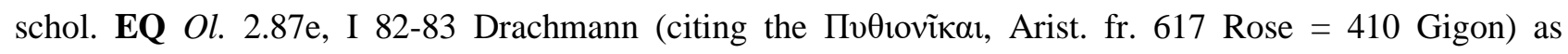
corrected by Rose (1863) 549 and Grassi (1961) 137 (ignored by Gigon).

${ }^{89}$ On the Isthmic and Nemean victor-lists see Christesen (2007) 108-111, arguing that the lists were incomplete and, at least in the case of the Isthmians, successive editions of the games were unnumbered. On the Isthmic records see also Gebhard (2002) 224.
}

90 'At that time neither the Corinthians nor the Argives had records concerning all the Nemean victors' (6.13.8). 
that at some unspecified later time both cities started keeping precisely such complete records, and that more piecemeal records existed earlier too. ${ }^{91}$ The headnote to Nemean 8 also suggests that Nemean records existed and survived for consultation but were not complete, at least with regard to

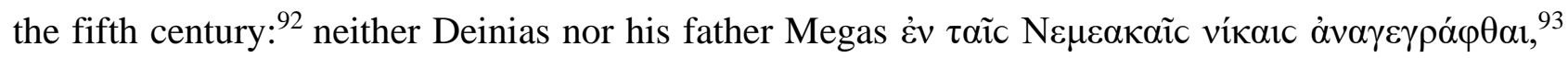
says Didymus (fr. 58 Braswell), whence the aporia on whether they won in the stadion or in the diaulos, since the text is indecisive. (The scholia seem to imply that the title went for the diaulos, see $* 46$ with $n$.) On the other hand, the headnote to Nemean 7 gives precise Nemead dates for Sogenes' victory and for the introduction of the pentathlon in the Nemean games. ${ }^{94}$ This is likely to come from a victor-list of some kind, or at any rate from a work concerned specifically with the Nemean games: the date by Nemeads - of which this is the only known example - is inexplicable otherwise.

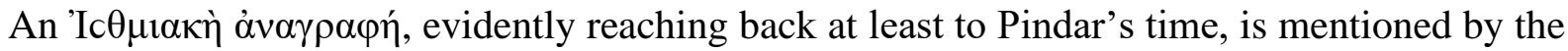
scholia to Isthmian 1, but the headnote to Olympian 12, at least in its surviving form, shows no awareness of a numbering of the Isthmiads. ${ }^{95}$ The headnote to Isthmian 3 deserves attention. It

${ }^{91}$ One would expect a reference to both Isthmian and Nemean winners, not only to the latter, whence a series

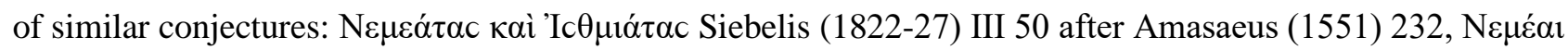

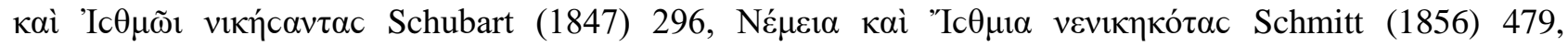

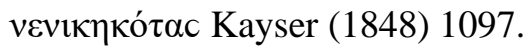

${ }^{92}$ Inscr. BD, III 140 Drachmann.

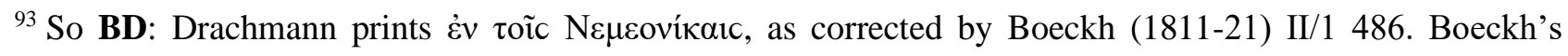

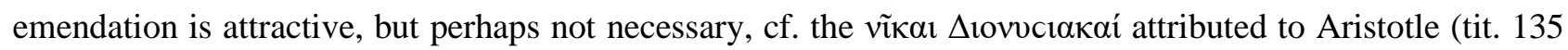
Gigon). In any case, a catalogue of Nemean victors is what the phrase must mean, see Christesen (2007) 108 n. 141.

${ }^{94}$ Inscr. BD, III 116 Drachmann.

${ }^{95}$ Schol. BD Isthm. 1.11c, III 199 Drachmann, inscr. a A Ol. 12, I 349 Drachmann; see Christesen (2007) 110. Plut. Ages. 21.5 is able to associate a specific list of victors with the Isthmian games of 390 , which were 


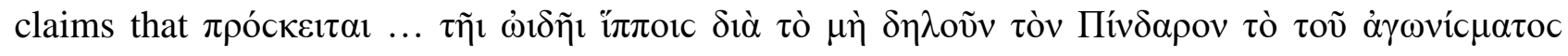

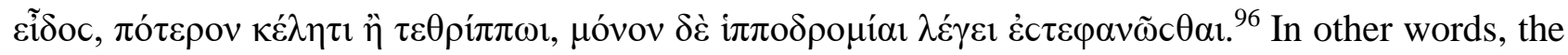
term ï $\pi \pi$ orc that originally stood in the title (see $* \mathbf{5 0}$ with $n$.) is alleged to be due to Pindar's own failure to specify which of the equestrian contests Melissos had won. The scholiast thus seems to have had no access to a victor-list that could provide the required clarification independently. ${ }^{97}$ But was he right to assume the same of whoever gave the ode its title? The word ï $\pi$ orc is found in three other Pindaric titles $(\mathbf{3 3}, \mathbf{3 9}, \boldsymbol{* 4 8})$, and in all three cases it refers to the chariot race. ${ }^{98}$ While the reason for alternating $\alpha \rho \mu \alpha \tau \iota$ and $i \pi \pi$ oic is unclear, the two words must refer to one and the same contest. If so, the fact that the title of Isthmian 3 specified the chariot race despite Pindar's ambiguity may suggest that a victor-list was consulted.

Nonetheless, given the paucity of the evidence, two further (and equally speculative) alternatives should be kept in mind. One is that the information may have come not from more or celebrated twice, but this very peculiarity may have made this Isthmiad identifiable in a way others perhaps were not. On the Plutarchean passage and its implications see Christesen (2007) 111-12 n. 152.

96 "The ode is introduced by "with horses" because Pindar does not clarify the kind of contest, whether (it was) with the single horse or with the four-horse chariot, but simply says that [Melissos] was crowned "in horse-racing"” (inscr. BD Isthm. 3, III 223 Drachmann).

${ }^{97}$ A few other scholiastic comments suggest ex silentio that a complete Isthmic victor-list was not available, by treating as ambiguous a matter that would not have been so otherwise: e.g. inscrr. a BD Nem. 11, b BD Isthm. 5, III 185, 241 Drachmann.

${ }^{98}$ In Pyth. 7 this will have been made clear by the Pythic victor-list, whereas in the other two poems it is explicitly stated in the text (Nem. 1.7, Isthm. 1.14). There may also be a fourth case, if the true reading in $\mathbf{1 6}$ is i $i \pi$ oıc not $\alpha \rho \mu \alpha \tau \imath$. There too it is clear that the chariot race was meant, since a victory in the mule cart race (the only alternative allowed by the text, see $\mathrm{n} .000$ ) could hardly be labelled i $\pi \pi$ orc. The variation between different forms with the same meaning had already been noted by Robert (1900) 145-46. Bacchylides has $i \pi \pi$ oıc twice $(\mathbf{8 8}, \mathbf{9 5}$; never $\alpha \rho \rho \alpha \tau \iota)$, and at least in $\mathbf{8 8}$ the word certainly refer to the chariot race (cf. 3.3-8). 
less complete festival records but from a more limited, local compilation of some sort. ${ }^{99}$ We have an example in $I G \mathrm{XII} / 5(1) 608$, a list of victorious athletes from Ceos probably dating from between 350 and $330,{ }^{100}$ which need not have been one of a kind. Otherwise, in Isthmian 3 as much as in Nemean 8, the lack of clarity on Pindar's part and of external guidance from a victor-list may have resulted in the editor making an arbitrary choice. The format of epinician titles required that the

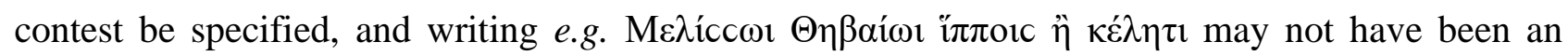
acceptable solution.

\section{Dithyrambs}

${ }^{99}$ Pace Fearn (2011b) 220-21, an existing record of Cean victors is a good explanation for Bacchylides' claim in 2.10-11 that Argeios' victory was the seventieth Isthmian 'garland' gained by Ceos: see Gelzer (1985) 99 (although we need not postulate an 'offizielle Siegerliste', as he does; any reckoning, official or otherwise, would do). The alternative - that immediately after the victory somebody went through the official records at the Isthmus, counted all the Cean victories, and found out that together with the new one they made a usefully round figure - does not sound quite as plausible. In the Panhellenic games Cean victors were proclaimed as Ceans, not as citizens of the individual Cean poleis, and ode 2 - which was probably performed at the Isthmus - makes no exception, as Fearn notes following Lewis (1962) 2. For all the complexities of Cean identity in the fifth century, it would be strange if the 'sentimental unity' (Lewis ibid.) of the island was exclusively limited to the Panhellenic stage, without as much as basic record-keeping at home. Furthermore, the supposed absence of such local records would require the fourth-century list of Cean victories in $I G \mathrm{XII} / 5(1) 608$ (see below) to have been compiled directly from the Isthmian and Nemean victor-lists, which in turn requires such lists to have been complete, contrary to what we have just seen.

${ }^{100}$ See Schmitt (1999), Fearn (2011b) 227-28; on the date, Schmitt (1999) 72-74. 
A second group of poems for which external evidence may be suggested is dithyrambs, which accounted for two volumes in the Alexandrian edition of Pindar and one in Bacchylides. ${ }^{101}$ Given the considerable diversity of original performance contexts and (presumably) of provenances in the corpus, one cannot expect all their titles to depend on similar material. Nonetheless, the possibility that some titles may have been based on material other than the bare texts is worth exploring.

Firstly, just as for athletic competitions, there were probably records of victories also at the competitions of the kyklioi khoroi at least in some parts of Greece. Athens springs to mind. Each year at the City Dionysia the ten tribes competed against one another with a chorus of men and one of boys. ${ }^{102}$ Competitions of 'circular choruses' took place at other Athenian festivals too. ${ }^{103}$ As concerned the dramatic competitions, the key facts of the Dionysia - poets, khorēgoi, plays, results, and the like - were duly recorded by the archon, and Aristotle published them in the form of a

${ }^{101}$ Pindar: Vita Ambrosiana I 3 Drachmann, P.Oxy. 2438 col. ii.36, cf. schol. BCEQ Ol. 13.25c, I 361 Drachmann with n. 000 above. Bacchylides: see Blass (1898) v-vi. According to the apparent practice of the ancient classifiers of late archaic lyric, or at any rate of Bacchylides' poems, I use the term 'dithyramb' as an equivalent of 'song performed by a circular chorus', with no strict Dionysiac implications. On the ancient terminology see Fearn (2007) 163-180, Wilson (2007) 164-69, further problematised by D'Alessio (2008) and (2013), Ceccarelli (2013).

${ }^{102}$ On the dithyrambic contest at the City Dionysia see Gould and Lewis in Pickard-Cambridge (19883) 7478, Ieranò (1997) 243-47.

${ }^{103}$ Anthesteria: Friis Johansen (1959), based on Copenhagen NM 13817 (ARV IX.57.35, 1145) and Pind. fr. 75 Snell-Maehler (but the latter can equally be related to the Dionysia, cf. Xen. Eq. mag. 3.2 with Deubner [1932] 140 n. 7, pace Friis-Johansen [1959] 41 n. 101); see also Gould and Lewis in Pickard-Cambridge $\left(1988^{3}\right)$ 16-17. Thargelia: Pickard-Cambridge $\left(1988^{3}\right) 75$ n. 2, 76 nn. 1, 3, Ieranò (1997) 247-49, Wilson (2000) 33-34, (2007) esp. 54-64, Fearn (2007) 235-38. Panathenaea: Ieranò (1997) 249-50, Wilson (2000) 39-40, Fearn (2007) 238-40. On Athenian dithyrambic performances see also Kowalzig and Wilson (2013) 13-18. 
catalogue known as Didaskaliai, 'Productions' (frr. 618-630 Rose, tit. 137 Gigon). ${ }^{104}$ The archon's records, whether mediated by Aristotle or not, also formed the basis of several inscriptions that were set up for display in Athens and elsewhere in the centuries that followed. ${ }^{105}$ Much like the Olympic and Pythic victor-lists in the study of epinician lyric, the Didaskaliai - 'a complete chronicle of performances of plays at the Dionysia and Lenaea' ${ }^{106}$ - were sedulously used by students of Attic drama, as the scholia to both tragedy and comedy testify. ${ }^{107}$ They could be used to date plays, to relate them to other plays produced on the same occasion, to assess their popularity with the judges, to trace and contextualise dramatists' careers, and (indirectly) to justify points of interpretation and literary history. But did they also include dithyrambs?

Rudolf Blum has denied this. ${ }^{108}$ As he notes, dithyrambs are not included in the inscribed $\Delta{ }_{1} \alpha c \kappa \alpha \lambda i \alpha_{1}$ and Victor-lists, which appear to have been extracts from Aristotle's Didaskaliai; dithyrambic victories are listed in the Fasti, which probably do not go back to Aristotle's work, ${ }^{109}$ but only the victorious tribe and its khorēgos are named, not the author or title of the winning dithyramb. Moreover, neither of the testimonies that are usually thought to imply the presence of dithyrambic poets in the Didaskaliai - Harpocration and a scholion to Aristophanes' Birds - states

${ }^{104}$ On the Didaskaliai see Rose (1863) 550-61, Jachmann (1909), Blum (1977) 50-92.

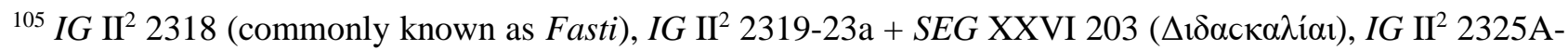
$\mathrm{H}$ (Victor-lists), and IGUR 215-16, 218. On the three Athenian inscriptions see now Millis and Olson (2012); on the Roman fragments - perhaps representing Callimachus' revision of Aristotle's Didaskaliai, see n. 000 - see Dittmer (1923).

${ }^{106}$ Blum (1977) 69, quoted from the English translation (p. 33).

${ }^{107}$ For a more sceptical take see Scullion (2002) 81-90; his argument, however, does not affect the use of didascalic records by more scholarly (or at least less credulous) sources than the playwrights' Lives, the Suda, and the like.

${ }^{108}$ Blum (1977) 52, 70-71.

${ }^{109}$ Koerte (1906) 392-3, see also Blum (1977) 68-69. 
explicitly that the respective poet, Pantacles and Cinesias, was recorded there as a

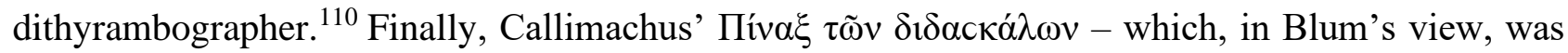
a mere reorganisation of Aristotle's Didaskaliai - is said by Choeroboscus to have contained $\alpha i$ àv $\alpha \gamma \rho \alpha \varphi \alpha i ̀ ~ . . \tau \tilde{\omega} \nu \delta \rho \alpha \mu \alpha ́ \tau \omega v$ 'the records of plays', with no mention of dithyrambs. ${ }^{111}$ But all these objections are open to doubt. Firstly, Harpocration and the scholiast state that Pantacles and Cinesias were referred to in the Didaskaliai: since neither dithyrambographer is also known to have been active as a tragic or comic poet, ${ }^{112}$ the natural implication is indeed that Aristotle included the dithyrambic contests in his records. Secondly, Choeroboscus - assuming that the Byzantine etymologist is reporting him accurately - said that Callimachus' didascalic register contained ('่v

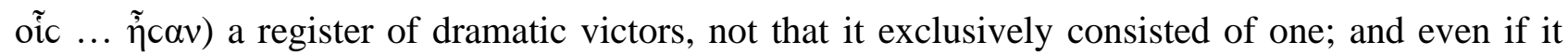
did, Callimachus may well have chosen to excerpt from Aristotle only the data relating to the dramatic compositions.

${ }^{110}$ Harp. $\delta 54$ Keaney, schol. VEГ Ar. Av. 1379b Holwerda (Arist. frr. 624, 629 Rose = 460, 445 Gigon); see Blum (1977) 70 with e.g. Jachmann (1909) 5-6, Pickard-Cambridge (1988³) 71.

${ }^{111}$ Callim. fr. 456 Pfeiffer ap. Etym. Magn. 1899 Gaisford; Blum (1977) 70.

${ }^{112}$ Blum (1977) 70 suggests that Cinesias and Pantacles may have also composed tragedies, but there is no solid evidence to support this view. Schol. VM@ Ar. Ran. 366c Chantry does make the extraordinary claim

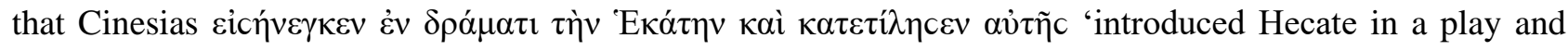
defecated all over her', but the text is unlikely to be genuine: the corresponding scholion in $\mathbf{R}$ (366b

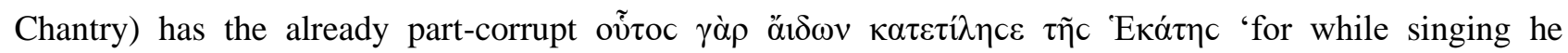

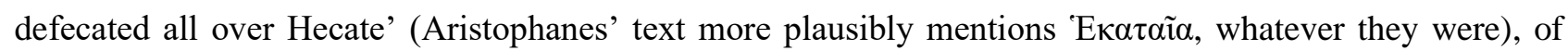
which $366 \mathrm{c}$ seems to be a misguided rationalisation. Cinesias is always referred to as a dithyrambographer, including in this very scholion, and the relevant line in Frogs explicitly locates the unfortunate episode

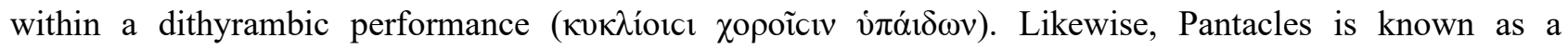
dithyrambic poet also from several inscriptions ( $\left.I G \mathrm{I}^{3} / 2958,959,967\right)$; see further Wilson (2007) 161. 
More importantly, if we look beyond Aristotle's name, there is further and more solid evidence that records of dithyrambic victories by named poets were available to later readers. The Marmor Parium (FGrHist 239 A 47, 49, 54, 65) mentions and dates the victories 'in Athens' of four poets: in 494/3 Melanippides of Melos (the grandfather of the New Musician of the same name), ${ }^{113}$ in $489 / 8$ a certain Simonides (allegedly, but improbably, the grandfather of the more famous Simonides of Ceos), ${ }^{114}$ Simonides of Ceos himself in 477/6, Telestes of Selinus in 402/1, and Polyidus of Selymbria sometime between 398/7 and 381/0. ${ }^{115}$ One cannot exclude derivation from a work on the history of music, or of poetry, or of Athenian theatrical competitions (but how far could a work of that kind have been itself independent of a victor-list?); but precisely such a victor-list is a plausible alternative. ${ }^{116}$ If the 'Simonidean' epigram 28 Page (FGE 796-801) is truly a post-classical product, ${ }^{117}$ the source of the punctiliously detailed information it contains - archon

\footnotetext{
${ }^{113}$ See Suda $\mu$ 454-55 Adler.
}

${ }^{114}$ Wilamowitz (1913) 139 n. 2 remarks the chronological unlikelihood of this relationship between the two

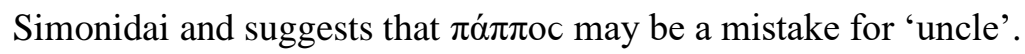

${ }^{115}$ On agonistic victories in the Marmor Parium and their Athenian slant see Rotstein (2016) 122-26.

${ }^{116}$ Rotstein (2016) 123, 125-26 distinguishes between information on early figures such as Thespis and

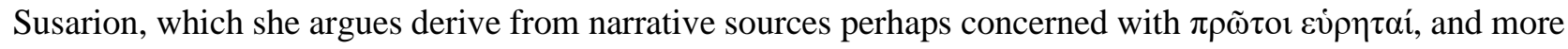
recent ones such as those mentioned above, which derive rather from didascalic records, 'perhaps ... used indirectly'.

${ }^{117}$ Simonidean authorship was first challenged by Stella (1946) 5-10, whose conclusions are accepted by Page in FGE ad loc. (arguing for a late Hellenistic date, pp. 242-43). Stella's objection that $\tau$ ic could not possibly be appended to the khorēgos' name (3) in an actual choregic monument is particularly hard to overrule; the rebuttal attempted by Wilson (2000) 369 n. 70 does not carry conviction, as David Sider points out (I am grateful to him for sharing with me the relevant chapter of his forthcoming commentary to the Simonidean epigrams). Sider inclines towards Simonidean authorship but takes the piece as a fragment of a 
(1. 1), tribe (2), khorēgos (3), division (4) and poet (5-6) - seems likely to have been a didascalic register. Finally, the author of the Life of Pindar preserved by P.Oxy. 2438 col. ii knows the date of one of Pindar's Athenian dithyrambs, which carried the prize in the archonship of Archias (497/6). While alternative explanations can no doubt be found, that of a list of dithyrambic victories seems the simplest. Aristotle's name may be the one most easily attached to such a list, but the identity of the compiler ultimately matters little.

A different question is whether such a list would have only included every year's winning dithyramb, as was the case for athletic victor-lists, or all those who competed. On the one hand, for tragedy and comedy the Didaskaliai did not limit themselves to victors, so if the dithyrambic records were also included in them, one would naturally expect a full list of contestants for those too. On the other hand, contrary to drama, much of the preparation for dithyrambic performances took place at tribe level, not at polis level. ${ }^{118}$ Although Blum's contention that the names of victorious poets were not recorded at all cannot be accepted, nevertheless it stands to reason that the authorities may only have recorded the only polis-wide datum, the victory. Furthermore, the number of dithyrambs that were performed every year greatly exceeded those of tragedies and comedies, and recording nine losers for every winner at the Dionysia alone may have been regarded as uneconomical. Like the other evidence examined at the end of the previous paragraph, P.Oxy. 2438 col. ii.8-10 cites a victory, not merely an entry. Since the anonymous biographer was sympotic elegy rather than as a self-standing public epigram, which may be a way to sidestep Stella's objection.

${ }^{118}$ Blum (1977) 71-72. Note, however, that poets seem to have been allocated to khorēgoi by lot, that is, centrally (Antiph. 6.11). Even if we take Antiphon's words to signify that khorēgoi 'drew lots for the order of choice between the available poets', as perhaps suggested by Ar. Av. 1404 and later testified by Dem. Meid. 13 for the choice of the aulos-player (Gould and Lewis in Pickard-Cambridge [19883 ] 75-56), the shortlist of poets to be picked must still have been compiled centrally: the procedure would have made little sense otherwise. 
evidently looking for the earliest date he could find in Pindar's career, the assumption that he (or his source) could consult a full list of contestants would require the inference that Pindar was victorious at his first attempt. ${ }^{119}$ We have two references to dithyrambic placements other than the first, in Isaeus (6.36) and Neanthes of Cyzicus the elder (BNJ $84 \mathrm{~F} \mathrm{10),} \mathrm{and} \mathrm{neither} \mathrm{of} \mathrm{them} \mathrm{seems} \mathrm{to} \mathrm{derive}$ from a didascalic record.

But another question is more relevant to this essay: whether and how far items in a dithyrambic didascalic list could be univocally related to existing poems. This means, for our purposes, whether such a list included the title - or $a$ title - for each dithyramb, whereby individual texts could be traced back to an individual festival and date. The parallel with the dramatic genres would suggest that titles were recorded, but the evidence fails to substantiate this suggestion. On the contrary, whereas at P.Oxy. 2438 col. ii.15-18 the biographer identifies what is apparently the latest Pindaric poem he could date (Olympian 4) by quoting its incipit, he offers no such identification for the Athenian dithyramb of 497/6. ${ }^{120}$ Being an argument from silence, this consideration has limited validity: the author's failure to identify the dithyramb explicitly need not mean that he could not have done so had he wished. It is also possible that the dithyramb in question had not survived to the biographer's day, or had an ambiguous title that did not allow it to be identified with certainty. Nonetheless, on the present state of our knowledge there is no proof that the dithyrambic victor-list allowed specific poems to be identified, and the one available piece of evidence seems rather to suggest otherwise.

Therefore, there is also no proof that the indication of the commissioning community found in some titles derives from external evidence. If we could be certain that $\mathbf{A}$ faithfully represents the ${ }^{119}$ Admittedly, nothing in the available evidence disproves this hypothesis, which may lie behind the anecdote reported by the Vita Ambrosiana, I 1 Drachmann: see Negri (2001) 1040. Alternatively, the biographer may only have been able to consult a shorter work that only listed winners, such as Aristotle's Nĩאal $\Delta$ tovvcıакаí (if these too included dithyrambic as well as dramatic contests).

${ }^{120}$ Noted by Arrighetti (1967) 132 n. 20. 
titles of the Alexandrian edition of Bacchylides' Dithyrambs, ${ }^{121}$ the absence of such indications from most titles would at least tell against the supposition that they were routinely fabricated. To relate ode 18 to Athens would have required no great effort of the imagination, and the conclusion that ode 17 was composed for the Ceans is all but demanded by its text (130-32); yet the relevant datives are not found on the manuscript $(\mathbf{9 9}, \mathbf{9 8})$. This would suggest that whenever the editor had no external documentation, he did not guess, but left a respectful blank. Tellingly, the only two titles where the commissioning community is indicated come from localities that we know had at least some victory records (100 from Athens, $\mathbf{1 0 1}$ from Sparta). ${ }^{122}$ However, seeing a definite correlation between the availability or otherwise of records and the indication or otherwise of the commissioning community in a title requires the supposition that ode 19 was performed at the Dionysia, odes 15 and 18 (probably also Athenian, but with no such indication in the title) at a festival other than the Dionysia, ${ }^{123}$ and ode 20 presumably at the Karneia. None of these hypotheses is new, ${ }^{124}$ but all are speculative, and defending the lot of them together would be a formidable task.

\footnotetext{
${ }^{121}$ On the problems raised by the two titling hands in $\mathbf{A}$ see pp. 000 above.

${ }^{122}$ The only relevant Spartan victory records we know of are that of the Karneia compiled by Hellanicus of Lesbos (BNJ 4 F 85a-86); on that work see Franklin (2010-11) and Pownall in BNJ ad loc. However, it is unclear whether dithyrambs were performed at the Karneia, see the next note.
}

${ }^{123}$ An alternative, if the didaskaliai only recorded victories, is that they were performed at the Dionysia but were not victorious.

${ }^{124}$ Ode 19 performed at the Dionysia: Fearn (2007) 181, 314 n. 174. Ode 15 performed at the Panathenaia: Zimmermann (1992) 69, Maehler (2004) 157-58, Fearn (2007) 240-41, 257-337. Ode 18 performed at the Thargelia: Jebb (1905) 235, Wilson (2007) 172-75. At the Theseia: Merkelbach (1973) 57. At the Hephaistia: Gelzer ap. Zimmermann (1992) 99 with n. 24. At the Panathenaia: Zimmermann (1992) 100. Ode 20 performed at the Karneia: Fearn (2007) 230 and n. 9, noting however that performances by kyklioi khoroi in that context are not documented with certainty; see now D'Alessio (2013) 123-24. At the festival 
At all events, too much uncertainty surrounds A's titles for any such argument to have much traction.

Whether any of the titles 'proper' go back to the respective authors, or at least to their time, is also difficult to answer without support from the Didaskaliai. ${ }^{125}$ Titles of dramatic pieces seemed to have been common currency relatively early, although not verifiably as early as the beginning of Pindar's career, even less of Simonides'. Alan Sommerstein argues 'that from the 470s at any rate, dramatic compositions were given titles by their authors which were recorded in the state archives; that from 446 or thereabouts, these titles were known to the public in advance of performance; that the titles by which plays were known in later antiquity and today are authentic in the overwhelming majority of cases; and that once the advance publication of titles began, dramatists started using them to mystify, tantalize and sometimes mislead their audiences as to what their play was going to be about'. ${ }^{126}$ It is tempting to imagine that the same went for dithyrambs, which later authors cited by title as a matter of course. However, no secure attestation of a dithyrambic title predates Aristotle, who mentions Timotheus' Scylla in the Poetics, Philoxenus' Banquet in a fragment of the

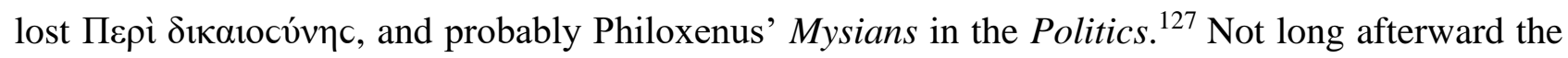
choregic inscription $I G \mathrm{II}^{2} 3055$ (dated to 320/19) records a reperformance of Timotheus' Elpenor. The latter piece must have been something of a classic by then; this may be the reason why the title was specified in the inscription, a unique occurrence among dithyrambic choregic dedications. ${ }^{128}$

of Artemis in Karyai: Zimmermann (1992) 105, see also Gołąb (2012). Another good candidate for the Panathenaia is ode $* * 23$ : Calame (2013) 82.

${ }^{125}$ A suggestion to this effect had been made by Lohan (1890) 19 after Bergk (1883) 535 n. 32; see also Ucciardello (1996-97) 72 with further bibliography at n. 25.

${ }^{126}$ Sommerstein (2002) 1, see also Kaimio (2000) 54-55 and already Lohan (1890) 19.

${ }^{127}$ Poet. 15, 1454a, fr. 83 Rose $=793$ Gigon, and Pol. 8, 1342b, where the mss. have $\pi$ oiñcal $\delta 1 \theta v ́ \rho \alpha \mu \beta o v$

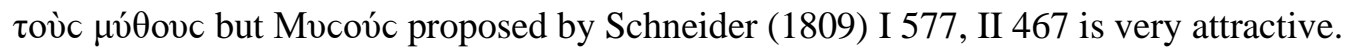

${ }^{128}$ Choregic inscriptions for dithyrambic victories are conveniently collected in Ieranò (1997) 331-61. 
Going further back in time than the mid-fourth century involves treading on very thin ice. A statement in Herodotus can be taken to say that the sixth-century citharode Arion of Methymna was the first to give a dithyramb a title, but an alternative interpretation is that he gave 'the' dithyramb its name, and no consensus has been reached. ${ }^{129}$ Nonetheless, the internal evidence provided by Bacchylides - since the Dithyrambs of Simonides and Pindar, like their titles, are too fragmentary to provide much guidance - bears exploring.

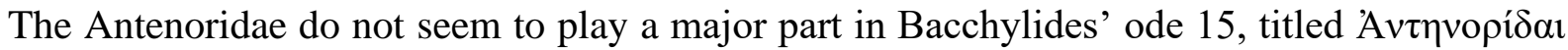

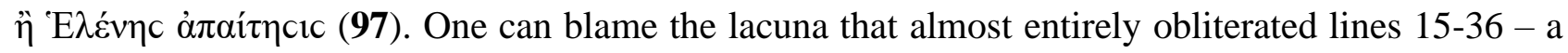
good third of the ode - for depriving us of a fulsome account of the gallantry they displayed in introducing Odysseus and Menelaus to the Trojan assembly; at least their number was probably mentioned, on the testimony of a scholion to the Iliad. ${ }^{130}$ But one is left with the feeling that titling the poem after the sons of Antenor would have been an odd choice for a later editor, who could have been content with the clearer and more informative The Demand for Helen's Return. Moreover, as Sir Richard Jebb realised, Antenor's fifty sons probably mirror the fifty choreutes of

${ }^{129}$ Hdt. 1.23, cf. Suda a 3886 Adler. See most recently D’Alessio (2013) 114, arguing for the second interpretation. The relevant section of the Herodotean passage runs $\delta 1 \theta \dot{\rho} \rho \alpha \mu \beta o v \pi \rho \tilde{\omega} \tau o v \dot{\alpha} v \theta \rho \omega ́ \pi \omega v \tau \tilde{\omega} v \dot{\eta} \mu \varepsilon \tilde{c}$

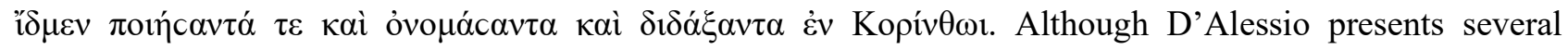
parallels for ỏvo $\mu \alpha \dot{\zeta} \omega$ applied to the naming of literary forms, two elements may also be taken to invite the interpretation of the verb as 'to title': the fact that in this passage both $\pi$ oińc $\alpha v \tau \alpha$ and $\delta 1 \delta \alpha \dot{\xi} \alpha \nu \tau \alpha$ are certainly particular (referring to the composition and production of a specific dithyramb, not of 'the dithyramb' as a

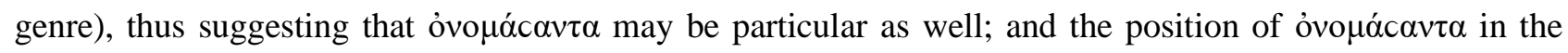
sequence, which arguably suits a reference to titling ('he composed a dithyramb, gave it a title, and produced it') more smoothly than to devising a name for the genre. But the matter is highly uncertain. If óvo $\mu \alpha \dot{\sigma} \sigma \alpha v \tau \alpha$ is indeed particular, it does not prove that dithyrambs truly had titles in Arion's time, but suggests that they did in Herodotus'.

${ }^{130}$ Schol. T Il. 24.496b Erbse. 
Athenian circular choruses, who will thus have visibly stood for them in performance. ${ }^{131}$ It is

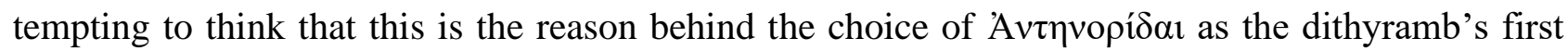
title. (The comparison with the many tragedies and comedies named after their respective choruses springs to mind.) If so, this choice seems likelier to have been made in the fifth century, either by Bacchylides himself or by someone in his extended audience, than several centuries later by someone wholly unconnected with the performance. ${ }^{132}$

Similar considerations can be made about the first title of ode 17 (98). The 'twice seven' are hardly the central figures of the story, although their mention perhaps specifies the episode in

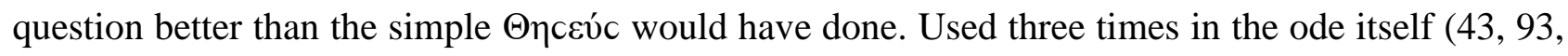
128), the word $\eta_{i} \theta \varepsilon$ co was almost a technical term for the fourteen Athenian youths sent to their death in Crete at Minos' behest. Interestingly, the Aristotelian Constitution of the Athenians applies the same term to the Athenian theōroi who were periodically sent to Delos to commemorate that mythical event (56.3). If scholars are right that the ode was performed by a Cean chorus on a mission to Delos, a hint of self-identification with the archetypal theōroi of the myth can be detected in the title as much as in the choice of theme and in the aetiological nod represented by the paean-singing at lines 128-29. ${ }^{133}$ If this is true, one feels once again that the title Youths is somewhat likelier to date from fifth-century Ceos (or Delos) than from second-century Alexandria. But proof remains to be found.

\footnotetext{
${ }^{131}$ Jebb (1905) 221, expanded by Zimmermann (1992) 68-69.

${ }^{132}$ Despite the differences, one may compare tragic titles like Aľac $\mu \alpha c \tau \imath \gamma о \varphi o ́ \rho o c, ~ ' I \pi \pi o ́ \lambda v \tau o c ~ \kappa \alpha \lambda v \pi \tau o ́ \mu \varepsilon v o c$,

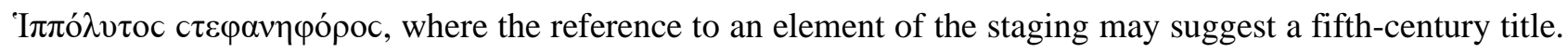
'Such names will not have originated with scholars who knew the plays mainly from reading but with people who knew them as spectacles': West (1979) 131.
}

${ }^{133}$ Similarly Ucciardello (1996-97) 73 n. 26. On the overlap between the chorus and the mythical Fränkel (1962) 515, Zimmermann (1992) 85; the aetiological implications of the paean-singing are highlighted by Calame (2009) 175, see already Rutherford (1997b) 48. 
The issue of alternative titles also requires investigation. Several Athenian dramas were known in antiquity with two titles joined by $\ddot{\eta}$, although with an interestingly different distribution between tragedy and comedy. ${ }^{134}$ The same is true of at least three lyric compositions regarded as dithyrambs by their ancient editors, the two of Bacchylides that we have just seen and one by Pindar (75): close to half of the dithyrambic titles known from these two authors (seven in total, plus the uncertain case of $\mathbf{1 0 1}$ to be discussed presently). What are the implication of this practice for the history of our texts? Sommerstein argues that double titles of Attic drama are not authorial and probably originated in the book trade. ${ }^{135}$ This is possible of dithyrambs too. Different manuscripts containing the same ode may have given it different titles. ${ }^{136}$ Perhaps, if titles were included in the dithyrambic victor lists after all, the didascalic title and the title given by one or more manuscripts may occasionally have been at variance. ${ }^{137}$ But a further possibility is that some alternative titles may result from the scholarly systematisazion that we know the poetry (and titles) of our three authors underwent. ${ }^{138}$

Unfortunately, the only piece of direct evidence on the subject - a passage from an anonymous commentary on (probably) Bacchylides' Dithyrambs - is lacunose and can only be restored conjecturally:

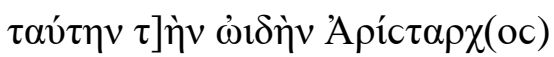

\footnotetext{
${ }^{134}$ Discussed in Sommerstein (2002) 5-8, with a convenient list at 14-16. See also Hunter (1983) 146-48.

${ }^{135}$ Sommerstein (2002) 5-6; see also Hunter (1983) 146, emphasising the role played by scholarly standardisation. Bender (1904) proposes a much later (and much less likely) date.

${ }^{136}$ Similarly Ucciardello (1996-97) 72 n. 25.

${ }^{137}$ Note, however, that none of the titles in question can compellingly be linked to a performance occasion in which the existence of didascalic records can be proved. Ode 15 is probably Athenian, but need not have been performed at the Dionysia: see p. 000 and n. 000 above.

${ }^{138}$ See again Ucciardello (1996-97) 72 n. 25.
} 


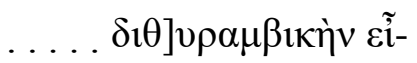

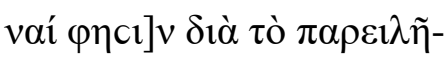

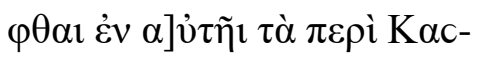

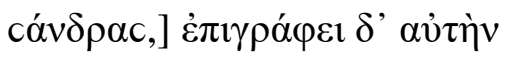

$\ldots \mathrm{K} \alpha c c] \alpha ́ v \delta \rho \alpha v$

(CLGP I.1.4 Bacchylides $4=$ B col. i.9-14)

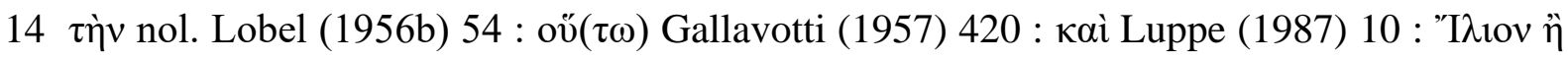
Luppe (1989) 29 : Siò D’Alessio (1997) 37 n. 90 : oũv Ucciardello (1996-97) 76 || cetera Lobel (1956b) 52

Aristarchus [says] that [this] ode is dithyrambic because the events concerning Cassandra are taken up [in] it, and [...] titles it Cassandra.

So Aristarchus was said to have given Bacchylides **23 a title, Cassandra (102). What is not obvious is whether this was an additional title of the kind we have just seen (supplying kaí with Luppe at line 14) $)^{139}$ or the ode's only one. The difficulty is compounded by uncertainty over whether Aristarchus was also the editor of the Alexandrian Bacchylides or only wrote a hypomnema

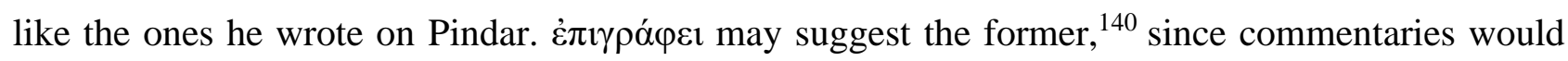
not seem to have normally been a place where to propose titles (even though some did report them). If so, what to supply at the beginning of line 14 is crucial for our understanding of titling practices. Aristarchus simply titling the ode Cassandra would be evidence that dithyrambic titles 'proper'

\footnotetext{
${ }^{139}$ It seems implausible that $\kappa \alpha$ in this context and position can be asseverative, as suggested by Käppel and Kannicht (1988) 21. If it is the correct supplement it must mean 'also'.

${ }^{140}$ So D’Alessio (1997) 53-54.
} 
could on occasion be entirely devised by editors, whether supplanting an earlier title or because the ode lacked one altogether. If instead he titled it 'also' Cassandra, the fact that the other title is not mentioned at all suggests that Aristarchus' was an addition to a pre-existing title - and that the reader, faced with this comment and with the double title itself (which will have been in the bookroll where the text of the poem was), could be expected to infer this essential piece of information without being told it explicitly. ${ }^{141}$

On the one hand, the hypothesis that Cassandra was the poem's only title sits well with the alphabetical order of Bacchylides' Dithyrambs. The first letter of the title cannot have been earlier than 1 , since the poem must have followed ode 20 (101). If Carlo Gallavotti was right to identify ode $* * 23$ with fr. dub. 60 Maehler, ${ }^{142}$ it also cannot have been later than $\lambda$, given the title of fr. dub. 61 Maehler (103) which follows it. On the other hand, none of the other supplements that have been proposed for the beginning of line 14 compares favourably to kaí. A whole further title is unlikely:

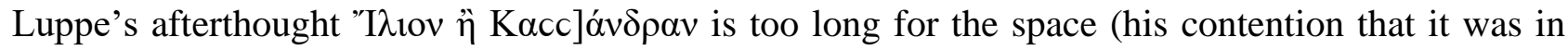
ekthesis has little to recommend it), ${ }^{143}$ and a suitable two-letter title to precede $\eta^{\prime \prime}$ is not easily found. A dislocated oũv (Ucciardello) is possible, ${ }^{144}$ but the unusual word-order tells against it somewhat. Other solutions are distinctly less attractive. On the whole, the less improbable reading seems to be

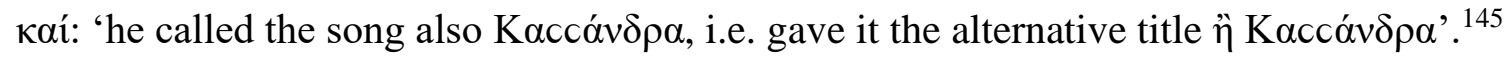

If this is true, then Aristarchus gave the poem an alternative title that was closely aligned to its subject-matter. While we are ignorant of what the first title was, the pattern is not unfamiliar. In ode

${ }^{141}$ For a contrary view see Hadjimichael (2014) 90 n. 36.

${ }^{142}$ Gallavotti (1950) 267, see now Hadjimichael (2014) 95-100.

${ }^{143}$ See in greater detail Ucciardello (1996-97) 71. It is possible, of course, that 'I $\lambda$ ıov (or perhaps 'I $\lambda \iota \alpha ́ \delta \varepsilon c$, which would suit fr. dub. 60 Maehler well) was the first title of the poem even though it was not mentioned at this point in the commentary.

${ }^{144}$ Parallels at Ucciardello (1996-97) 76.

${ }^{145}$ Luppe (1987) 10-11. 
15, as we saw, a meaningful but somewhat unperspicuous first title was clarified by the second (97). Perhaps a similar argument can be made with reference to ode 17 (98). We do not know a great deal about Pindar's second Dithyramb, ${ }^{146}$ but the addition of Ké $\rho \beta \varepsilon \rho o c$ clarifies the subject-matter of the narrative - Heracles' descent to Hades - better than the plain 'H $\rho \alpha \kappa \lambda \tilde{\eta} c$ (75). This raises the interesting possibility that at least some narrative lyric poems were already furnished with a title perhaps, though not necessarily, going back as far as the fifth century - when they reached their editor, whereupon some of them received an additional one for clarification or completeness. But it is also possible that some poems reached Alexandria from two different sources and with two different titles, or that an editor could not make up his mind which of two titles of his own invention was more appropriate for a given poem. Moreover, the editors of the respective poets were not the only scholars who could assign titles. Callimachus' monumental Pinakes were a work to be reckoned with, as demonstrated by Aristarchus' refutation of his classification of Bacchylides **23

in B. Other scholars too could refer to texts in non-canonical ways. ${ }^{147}$ More importantly, when a poem only has one title in the relevant "standard" edition, it is impossible to assess whether it was an existing title with which the editor was satisfied, or the poem had reached the Library without a title and the editor then supplied one.

\section{Conclusions}

As is all too frequently the case, it is impossible to reconstruct with exactitude how the evidence we have came into being. The section dedicated to Pindar's Epinicians has shown that independent evidence was consistently used when available. Our examination of Bacchylides'

\footnotetext{
${ }^{146}$ Note, however, the substantial improvement on earlier texts achieved by Lavecchia (1996) and (2000) 3031, 106-09, who makes a strong case for also recognising fr. 346a-c Snell-Maehler as a part of Dithyramb 2.

${ }^{147}$ See the passages collected by Hadjimichael (2014) 93 n. 48, esp. Ath. 11.496e-f.
} 
Dithyrambs has tentatively suggested that at least in certain cases pre-existing data could be retained, although not necessarily unmodified. (The only datum we know with a good degree of plausibility is that the title Cassandra in $\mathbf{1 0 1}$ is a scholar's invention.) What we can glimpse is a picture of reasonable accuracy within the limits of Hellenistic scholarly practice and of the evidence that Aristophanes of Byzantium and his colleagues had at their disposal. Sources of relevant material were mined and put to use. Although occasionally guesswork will have been inevitable, it could also be eschewed in favour of an (implicit) admission of ignorance: contrary to the standard format for titles of Paeans, the dative indicating the commissioning community was not included in 69. ${ }^{148}$

This raises the possibility that material other than the bare texts of the poems could be resorted to even when we no longer have evidence for its use. Such a possibility cannot be demonstrated, much less determined or quantified. Nevertheless, it can be usefully taken into consideration on a general level. Possible examples (if we accept the possibility that the disunity of Paean 6 predates in some form the "standard" Pindar) are 64 and 65 (=73?): the notion of a Delphic commission is not obviously supported by the substantial extant remains of Paean $6(\mathrm{a}),{ }^{149}$ and nothing in the opening or closing section of Paean 6(b) suggests a specific link to a cult of Aeacus. But other explanations for these indications can be hypothesised. The claim made in $\mathbf{6 4}$ may simply result from the lack of specific reference to a non-Delphic origin of the chorus in what is an ostentatiously Delphic milieu; that made in $\mathbf{6 4}$ may depend on the editor's awareness of the Aiakeia

\footnotetext{
${ }^{148}$ The presence of the word $\pi \alpha$ lóv in its stead guarantees that its absence is not a case of scribal omission. Nor can it be ascribed to scribal idiosyncrasy, given that $\mathbf{6 7}$ on the same manuscript has the usual format.

${ }^{149}$ Indeed, that the performers of the united Paean 6 were Delphian has been doubted by several scholars in modern times, starting with Wilamowitz (1908) 350 almost immediately upon the publication of $\Pi^{4}$; for further bibliography see Currie (2005) 323 n. 143. Of course the lacuna that obliterated half of the first triad may well be to blame for the loss of any further information on the commission of the poem.
} 
in Aegina more than of a specific connexion between that festival and Paean 6(b). Pindar's dictum seems once again most appropriate:

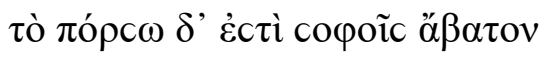

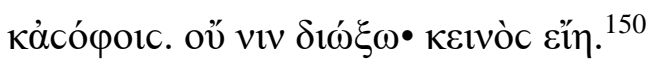

150 'What lies beyond neither wise men nor fools can tread. I will not pursue it; it would be foolish' (Pind. Ol. 3.44-45). 


\section{Bibliography}

Amasaeus, R. 1551. Pausaniae veteris Graeciae descriptio. Florence.

Arrighetti, G. 1967. La biografia di Pindaro del papiro di Ossirinco XXVI 2438. SCO 16: 129-48.

Barrett, W. S. 2007. Pindar and Psaumis: Olympians 4 and 5. In Greek Lyric, Tragedy, Collected Papers, ed. M. L. West, 38-53. Oxford.

Bender, G. 1904. De Graecae comoediae titulis duplicibus. Marburgi Cattorum.

Bergk, Th. 1866-1867. Poetae lyrici Graeci. Vols 1-3. $3^{\text {rd }}$ edition. Leipzig.

Bergk, Th. 1878-82. Poetae lyrici Graeci. Vols 1-3. $4^{\text {th }}$ edition. Leipzig.

Bergk, Th. 1883. Griechische Literaturgeschichte. Vol. 2, ed. G. Hinrichs. Berlin.

Blass, F. 1898. Bacchylidis carmina cum fragmentis. Leipzig.

Blum, R. 1977. Kallimachos und die Literaturverzeichnung bei den Griechen. Untersuchungen zur Geschichte der Biobibliographie. Frankfurt am Main [English translation: H. H. Wellisch. 1991. Kallimachos. The Alexandrian Library and the Origins of Bibliography. Madison WI].

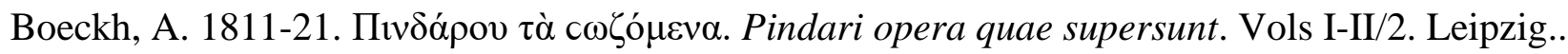
Borgo, A. 2007. Quando il libro si presenta da sé: Arma virumque e i titoli delle opera antiche. Aevum 81: 133-47.

Braswell, B. K. 1988. A Commentary on the Fourth Pythian Ode of Pindar. Berlin and New York. Breitenbach, H. 1908. De genere quodam titulorum comoediae Atticae. Basle.

Budin, S. L. 2008. The Myth of Sacred Prostitution in Antiquity. Cambridge.

Calame, C. 2009. Apollo in Delphi and in Delos: Poetic Performances between Paean and Dithyramb. In Apolline Politics and Poetics, eds. L. Athanassaki, R. P. Martin, and J. F. Miller, 169-97. Athens.

Calame, C. 2013. "Rien pour Dionysos?" Le dithyrambe comme forme poétique entre Apollon et Dionysos. In Redefining Dionysos, eds. A. Bernabé, M. Herrero de Jáuregui, A. I. Jiménez San Cristóbal, and R. Martín Hernández, 82-99. Berlin and Boston.

Carey, C. 1983. Review of Maehler (1982). JHS 103: 165-66. 


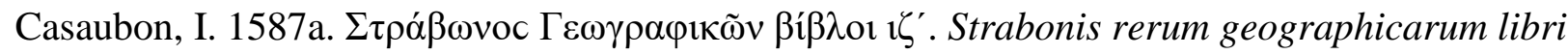
XVII. [Geneva].

1587b. Commentarius et castigationes ad lib. Strabonis geograph. XVII. [Geneva].

Cavallo, G. 1974. Lo stile di scrittura 'epsilon-theta' nei papyri letterari. CErc 4: 33-36. Reprinted in Il calamo e il papiro. La scrittura greca dall'età ellenistica ai primi secoli di Bisanzio, 123-28. Florence 2005.

Ceccarelli, P. 2013. Circular Choruses and the Dithyramb in the Classical and Hellenistic Period. A Problem of Definition. In Dithyramb in Context, eds. B. Kowalzig and P. Wilson, 153-70. Oxford.

Christesen, P. 2007. Olympic Victor Lists and Ancient Greek History. Cambridge.

Currie, B. G. F. 2005. Pindar and the Cult of Heroes. Oxford.

D’Alessio, G. B. 1997. Pindar's Prosodia and the Classification of Pindaric Papyrus Fragments. ZPE 118: 23-60.

2000. Review of S. Schröder (1999). BMCR 2000.01.24. 2001. Sulla struttura del libro dei Peani di Pindaro. In I lirici greci. Forme della comunicazione e storia del testo, eds. M. Cannatà Fera and G. B. D’Alessio, 69-86. Messina. 2004. Argo e l'Argolide nei canti cultuali di Pindaro. In La città di Argo. Mito, storia, tradizioni poetiche. Atti del convegno internazionale (Urbino, 13-15 giugno 2002), ed. P. Angeli Bernardini, 107-25. Rome. 2008. Review of Fearn (2007). BMCR 2008.11.14. 2012. The Lost Isthmian Odes of Pindar. In Reading the Victory Ode, eds. P. Agócs, C.

Carey, and R. Rawles, 28-57. Cambridge. 2013. "The Name of the Dithyramb". Diachronic and Diatopic Variations. In

Dithyramb in Context, eds. B. Kowalzig and P. Wilson, 115-32. Oxford. 2016. Bacchylides' Banquet Songs. In The Cup of Song. Studies on Poetry and the Symposion, eds. V. Cazzato, D. Obbink and E. E. Prodi, 63-84. Oxford. 
D’Alessio, G. B. and Ferrari, F. 1988. Pindaro, Peana 6, 175-183: una ricostruzione. SCO 28: 15980.

Daude, C. et al. 2013. Scholies à Pindare, I: Vies de Pindare et scholies à la première Olympique. Besançon.

Davison, J. 1934. The Authorship of the 'Leucippides' Papyrus. CR 48: 205-07.

Del Mastro, G. 2014. Titoli e annotazioni bibliologiche nei papiri greci di Ercolano. Naples.

De Sanctis, G. 1928. Lacare. RFIC NS 6: 53-77. Reprinted in Scritti minori. Vol. 1, 349-69, Rome 1966.

Deubner, L. 1932. Attische Feste. Berlin.

Dittmer, W. A. 1923. The Fragments of the Athenian Comic Didascaliae Found in Rome (IG XIV 1097, 1098, 1098a). Leiden.

Duchet, C. 1973. "La fille abandonee" et "La bête humaine". Éléments de titrologie romanesque. Littérature 12: 49-73.

Fearn, D. 2007. Bacchylides. Politics, Performance, Poetic Tradition. Oxford.

Fearn, D. 2009. Oligarchic Hestia: Bacchylides 14B and Pindar, Nemean 11. JHS 129: 23-38.

Fearn, D. 2011a. Aeginetan Epinician Culture: Naming, Ritual, and Politics. In Aegina: Contexts for Choral Lyric Poetry. Myth, History, and Identity in the Fifth Century BC, ed. D. Fearn, 175-226. Oxford.

Fearn, D. 2011b. The Ceians and their Choral Lyric: Athenian, Epichoric and Pan-Hellenic Perspectives. In Archaic and Classical Choral Song. Performance, Politics and Dissemination, eds. L. Athanassaki and E. L. Bowie, 207-34. Berlin and New York. Ferry, A. 1996. The Title to the Poem. Stanford.

Filoni, A. 2007. Il peana di Pindaro per Dodona (frr 57-60 M.). Milan.

Finglass, P. J. 2007. Pindar. Pythian Eleven. Cambridge.

Fränkel, H. 1962. Dichtung und Philosophie des frühen Griechentums. Eine Geschichte der griechischen Epik, Lyrik und Prosa bis zur Mitte des fünften Jahrhunderts. $2^{\text {nd }}$ edition. 
Munich. [English translation: M. Hadas and J. Willis, trans., Early Greek Poetry and Philosophy. A History of Greek Epic, Lyric, and Prose to the middle of the fifth century. Oxford 1975.]

Franklin, J. C. 2010-11. The Lesbian singers: towards a reconstruction of Hellanicus' Karneian Victors. Rudiae 22-23: II 719-63.

Fredouille, J.-C. et al. 1997. Titres et articulations du texte dans les œuvres antiques. Actes du Colloque International de Chantilly 13-15 décembre 1994, eds. J. C. Fredouille, M.-O. Goulet-Cazé, Ph. Hoffmann, and P. Petitmengin. Paris.

Friis Johansen, K. 1959. Eine Dithyrambos-Aufführung. København.

Gallavotti, C. 1950. Review of Bacchylidis carmina cum fragmentis, ed. B. Snell, Leipzig $1949^{6}$. RFIC NS 28: 265-67.

Gallavotti, C. 1957. Review of The Oxyrhynchus Papyri 23 (1956). Gnomon 29: 419-25.

Gallo, I. 1968. Una nuova biografia di Pindaro (POxy. 2438). Introduzione, testo critico e commentario. Salerno.

Gebhard, E. R. 2002. The beginnings of Panhellenic games at the Isthmus. In Olympia 1875-2000: 125 Jahre deutsche Ausgrabungen. Internationales Symposion, Berlin 9.-11. November 2000, ed. H. Kyrieleis, 221-37. Mainz am Rhein.

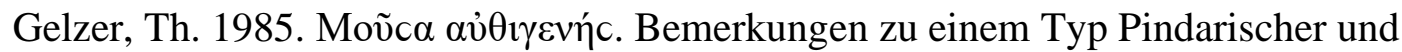
Bacchylideischer Epinikien. $M H$ 42: 95-120.

Genette, G. 1987. Seuils. Paris. [Engl. transl. J. E. Lewin, Paratexts. Thresholds of Interpretation. Cambridge 1997.]

Genette, G. 1988. Structure and function of the title in literature. Critical Inquiry 14: 692-720.

Gentili, B. et al. 2012. Pindaro. Le Pitiche. $5^{\text {th }}$ edition. Milan.

Gołąb, H. 2012. Bacchylides' Spartan Dithyramb in the Light of Choral Projection. Eos 99: 15-22.

Grassi, E. 1961. Inediti di Eugenio Grassi. $A \& R$ NS 6: 129-65. 
Grenfell, B. P. and Hunt, A. S. 1899. CCXXII List of Olympian victors. The Oxyrhynchus Papyri 2: $85-95$.

1903. 408 Odes of Pindar. The Oxyrhynchus Papyri 3: 13-17. 1908. 841 Pindar, Paeans. The Oxyrhynchus Papyri 5: 11-110.

Hadjimichael, Th. 2014. Fr. 60 M. of Bacchylides and the Kassandra. BASP 51: 77-100.

Hippenstiel, G. 1887. De Graecorum tragicorum principum fabularum nominibus. Marburg.

Hoek, L. H. 1981. La marque du titre. Dispositifs sémiotiques d'une pratique textuelle. La Haye, Paris and New York.

Holtz, L. Titre et incipit. In Fredouille et al. 1997: 469-89.

Houston, G. W. 2014. Inside Roman Libraries. Book Collections and their Management in Antiquity. Chapel Hill.

Hunt, A. S. 1927. 2082 Phlegon, Chronica (?). The Oxyrhynchus Papyri 17: 82-99.

Hunter, R. L. 1983. Eubulus. The Fragments. Cambridge.

Ieranò, G. 1997. Il ditirambo di Dioniso. Le testimonianze antiche. Pisa and Rome.

Irigoin, J. 1952. Histoire du texte de Pindare. Paris.

Irigoin, J., Duchemin, J. and Bardollet, L. 1993. Bacchylide. Dithyrambes - Épinicies - Fragments. Paris.

Jachmann, G. 1909. De Aristotelis didascaliis. Göttingen.

Jebb, R. C. 1905. Bacchylides. The Poems and Fragments. Cambridge.

Kaimio, M. 2000. Tragic Titles in Comic Disguises. In Studies in Ancient Literary Theory and Criticism, ed. J. Styka, 53-69. Kraków.

Käppel, L. and Kannicht, R. 1988. Noch einmal zur Frage ‘Dithyrambos oder Paian?’ im Bakchylideskommentar P.Oxy. 23.2368. ZPE 73: 19-24.

Kayser, K. L. 1848. Zur Kritik des Pausanias. JfA 6: 495-511, 997-1007, 1079-1101.

Kenyon, F. G. 1897. The Poems of Bacchylides from a Papyrus in the British Museum. Oxford.

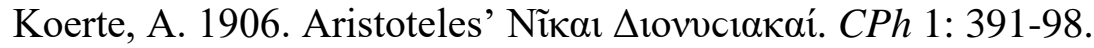


Komorzynski, E. von. 1903-1904. Zur Geschichte der Blume im deutschen Buchtitel. Zeitschrift für Bücherfreunde 7: 284-87.

Kowalzig, B. and Wilson, P. 2013. Introduction: The World of Dithyramb. In Dithyramb in Context, 1-27. Oxford.

Kowerski, L. M. 2005. Simonides on the Persian Wars. A Study of the Elegiac Verses of the 'New Simonides'. New York and London.

Kurke, L. 2007. Visualizing the Choral: Epichoric Poetry, Ritual, and Elite Negotiation in FifthCentury Thebes. In Visualizing the Tragic. Drama, Myth, and Ritual in Greek Art and Literature. Essays in honour of Froma Zeitlin, eds. C. Kraus et al., 63-102. Oxford.

Lavecchia, S. 1996. P. Oxy. 2622 e il 'Secondo Ditirambo' di Pindaro. ZPE 110: 1-26.

Lavecchia, S. 2000. Pindari dithyramborum fragmenta. Rome and Pisa.

Lehnus, L. 1973a. Una glossa pindarica in Corinna di Tanagra. RIL 107: 393-422.

Lehnus, L. 1973b. Contributo a due frammenti pindarici (frr. 37 e 168 Snell $^{3}$ ). SCO 22: 5-18.

Lehnus, L. 1984. Pindaro: il dafneforico per Agasicle (Fr. 94b Sn.-M.). BICS 31: 61-92.

Levenston, E. A. 1978. The Significance of the Title in Lyric Poetry. The Hebrew University Studies in Literature 6: 63-87.

Levin, H. 1977. The Title as a Literary Genre. The Modern Language Review 72: xxiii-xxxvi. Lewis, D. M. 1962. The Federal Constitution of Keos. ABSA 57: 1-4.

Lobel, E. 1956a. 2366 Bacchylides, "Y Yvor? The Oxyrhynchus Papyri 23: 40-41. 1956b. 2368 Commentary on Bacchylides?, Dithyrambs or Paeans. The Oxyrhynchus Papyri 23: 51-54. 1959a. 2430 Choral lyric in the Doric dialect (?Simonides). The Oxyrhynchus Papyri 25: $45-87$ 1959b. 2431 Simonides, Epinicians? The Oxyrhynchus Papyri 25: 87-94. 1968. 2734 On Alcaeus. The Oxyrhynchus Papyri 35: 2-8. 
Lohan, E. 1890. De librorum titulis apud classicos scriptores Graecos nobis occurrentibus.

Marburg.

Luppe, W. 1987. Dithyrambos oder Paian? - Zu Bakchylides Carm. 23 Sn./M. ZPE 69: 9-12. 1989. Nochmals zu Kallimachos’ Gattungszuordnung bakchylideischer Lieder.

APapyrol 1: 23-29.

Maehler, H., ed. 1982. Die Lieder des Bakchylides. Vol. 1. Die Siegeslieder. Leiden. ed. 1989. Pindari carmina cum fragmentis. Vol. 2: Fragmenta, indices. $8^{\text {th }}$ edition.

Leipzig. 2004. Bacchylides. A Selection. Cambridge.

Merkelbach, R. 1973. Der Theseus des Bakchylides (Gedicht für ein attisches Ephebenfest). ZPE 12: $56-62$

Millis, B. W. and Olson, S. D. 2012. Inscriptional Records for the Dramatic Festival in Athens. IG II 2318-2325 and Related Texts. Leiden and Boston.

Mommsen, C. I. T., ed. 1864. Pindari carmina. Berlin.

Nachmanson, E. 1969. Der griechische Buchtitel. Einige Beobachtungen. Darmstadt.

Negri, M. 2001. La trattazione della cronologia pindarica nel POxy XXVI 2438. In Atti del XXII Congresso Internazionale di Papirologia. Firenze, 23-29 agosto 1998, eds. I. Andorlini, G. Bastianini, M. Manfredi and G. Menci, vol. 2: 1033-43. Florence.

Negri, M. 2004. Pindaro ad Alessandria. Le edizioni e gli editori. Brescia.

Obbink, D. 2001. The Genre of Plataea. Generic Unity in the New Simonides. In The New Simonides. Contexts of Praise and Desire, eds. D. Boedeker and D. Sider, 65-85. New York. Parsons, P. 1992. 3965 Simonides, Elegies. The Oxyrhynchus Papyri 59: 4-50.

Paton, W. R. 1912. Simonides, fr. 68, and a fragment of Lupercus. CR 26: 9.

Pickard-Cambridge, A. 1988. The Dramatic Festivals of Athens. Second edition revised by J. Gould and D. M. Lewis, reissued with supplement and corrections. Oxford. 
Poltera, O. 2005. Deliaka (Simon. PMG 539): zu einer vermeintlichen Gedichtsammlung. SIFC s. IV 3: 183-87.

Poltera, O. 2008. Simonides Lyricus. Testimonia und Fragmente. Basle.

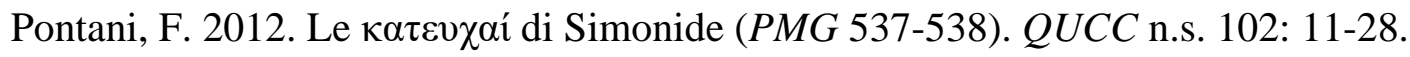

Porro, A. 1994. Vetera Alcaica. L'esegesi di Alceo dagli Alessandrini all'età imperiale. Milan.

Prodi, E. E. 2013. P.Oxy. 2448 (Pi. fr. 215 Sn.-M.) and Pindar's Prosodia. ZPE 185: 53-59. 2014. A Bibliological Note on P.Oxy. 659 (Pindar, Partheneia). APapyrol 26: 99-105. 2016. Titles and Markers of Poem-end in the Papyri of Greek Choral Lyric. In

Proceedings of the XXVII International Congress of Papyrology, eds. T. Derda, A. Łajtar, and J. Urbanik, 1137-1184. Warsaw.

Race, W. H. 1997. Pindar. Vols 1-2. Cambridge MA and London.

Rawles, R. 2013. Aristophanes' Simonides: Lyric Models for Praise and Blame. In Greek Comedy and the Discourse of Genres, eds. E. Bakola, L. Prauscello, and M. Telò, 175-201. Cambridge.

Robert, C. 1900. Die Ordnung der olympischen Spiele und die Sieger der 75.-83. Olympiade. Hermes 35: 141-195.

Rose, V., ed. 1863. Aristoteles pseudepigraphus. Leipzig.

Rose, V., ed. 1886. Aristotelis qui ferebantur librorum fragmenta. Leipzig.

Rotstein, A. 2016. Literary History in the Parian Marble. Washington DC.

Rutherford, I. C. 1988. Pindar on the Birth of Apollo. $C Q$ n.s. 38: 65-75. 1990. Paeans by Simonides. HSCPh 93: 169-209. 1995. Et hominum et deorum ... laudes (?): A hypothesis about the Organisation of Pindar's Paean-book. ZPE 107: 44-52. 1997a. For the Aeginetans to Aeacus a prosodion: An Unnoticed Title at Pindar, Paean 6, 123, and its significance for the poem. ZPE 118: 1-21. 
1997b. Odes and ends: closure in Greek lyric. In Classical Closure. Reading the End in

Greek and Latin Literature, eds. D. H. Roberts, F. M. Dunn, and D. Fowler, 43-61. Princeton. 2001a. Pindar's Paeans. A Reading of the Fragments with a Survey of the Genre.

Oxford.

2001b. The New Simonides. Toward a Commentary. In The New Simonides. Contexts

of Praise and Desire, eds. D. Boedeker and D. Sider, 33-54. New York.

forthcoming. Simonides, Anius and Athens. A note on PMG 537 (Kateukhai). In

Simonides lyricus, eds. P. Agócs and L. Prauscello. Cambridge.

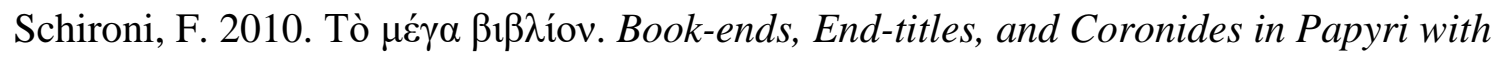
Hexametric Poetry. Durham NC.

Schironi, F. 2016. 5277 Oppian, Halieutica 4.683-93. The Oxyrhynchus Papyri 81: 85-87.

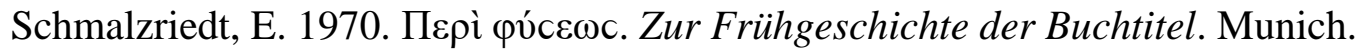

Schmidt, D. 1999. An Unusual Victory List from Keos: IG XII, 5, 608 and the Dating of Bakchylides. JHS 119: 67-85.

Schmitt, J. C. 1856. Beiträge zur kritik des Pausanias. Philologus 11: 468-79.

Schneider, I. G. 1809. Aristotelis Politicorum libri octo superstites. Vols. 1-2. Frankfurt a.d. Oder.

Schneidewin, F. W. 1846. De Peplo Aristotelis Stagiritae. Accedunt Pepli reliquiae. Philologus 1: $1-45$.

Schröder, B.-J. 1999. Titel und Text. Zur Entwicklung lateinischer Gedichtsüberschriften. Mit Untersuchungen zu lateinischen Buchtiteln, Inhaltsverzeichnissen und anderen Gliederungsmitteln. Berlin and New York.

Schröder, S. 1999. Geschichte und Theorie der Gattung Paian. Eine kritische Untersuchung mit einem Ausblick auf Behandlung und Auffassung der lyrischen Gattungen bei den alexandrinischen Philologen. Stuttgart and Leipzig.

Schubart, J. H. C. 1847. Beiträge zur Kritik des Pausanias. JfA 5: 217-23, 225-30, 289-300.

Scullion, S. 2002. Tragic dates. CQ n.s. 52: 81-101. 


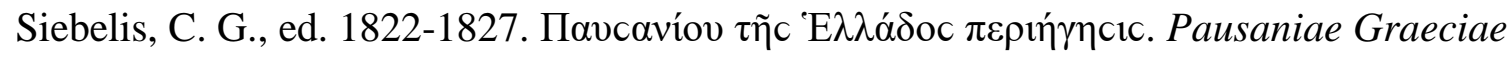
descriptio. Vols 1-4. Leipzig.

Snell, B., ed. 1934. Bacchylidis carmina cum fragmentis. $5^{\text {th }}$ edition. Leipzig. 1937. Euripides Alexandros und andere strassburger Papyri mit Fragmente griechischer Dichter. Berlin. , ed. 1958. Bacchylidis carmina cum fragmentis. $7^{\text {th }}$ edition. Leipzig. 1964. Pindari carmina cum fragmentis. Vol. 2: Fragmenta, indices. $3^{\text {rd }}$ edition. Leipzig. 1965. Dichtung und Gesellschaft. Studien zum Einfluß der Dichter auf das soziale Denken und Verhalten im alten Griechenland. Hamburg

Sommerstein, A. H. 2002. The Titles of Greek dramas. SemRom 5: 1-16. Reprinted with addenda in The Tangled Ways of Zeus and Other Studies in and around Greek Tragedy, 11-29. Oxford, 2002.

Spoerri, W. 1988. Epigraphie et littérature: à propos de la liste des Pythioniques à Delphes. In Comptes et inventaires dans la cite grecque. Actes du colloque international d'épigraphie tenu à Neuchâtel du 23 au 26 septembre 1986 en l'honneur de Jacques Tréheux, ed. D. Knoepfler, 111-40. Neuchâtel and Geneva.

Stella, L. A. 1946. Studi simonidei. RFIC NS 24: 1-24.

Turyn, A., ed. 1944. Pindari epinicia. New York.

Turyn, A., ed. 1948. Pindari carmina cum fragmentis. Kraków.

Ucciardello, G. 1996-97. Riesame di P. Oxy. 2368: alcuni problemi di lettura e di interpretazione. APapyrol 8-9: 61-88.

Ucciardello, G. 2016. New Light on P.Strasb. gr. 1406-1409: An Early Witness of Secundus' Sentences. In Proceedings of the XXVII International Congress of Papyrology, eds. T. Derda and J. Urbanik, 251-57. Warsaw.

Vogliano, A. 1932. Frammenti di poemetti lirici. Papiri greci e latini 10: 169-79. 
West, M. L. 1979. The Prometheus Trilogy. JHS 99: 130-48. Reprinted in Hellenica. Selected Papers on Greek Literature and Thought. Vol 2, 250-86. Oxford 2013.

Wilamowitz-Moellendorff, U. von. 1908. Pindars siebentes nemeisches Gedicht. SPAW 1908: 32852. Reprinted in Kleine Schriften. Vol. 6, 286-313. Berlin 1972.

Wilamowitz-Moellendorff, U. von. 1913. Sappho und Simonides. Untersuchungen über griechische Lyriker. Berlin.

Wilamowitz-Moellendorff, U. von. 1922. Pindaros. Berlin.

Wilson, P. 2000. The Athenian Institution of Khoregia. The Chorus, the City and the Stage. Cambridge.

Wilson, P. 2007. Performance in the Pythion: the Athenian Thargelia. In The Greek Theatre and Festivals. Documentary Studies, ed. P. Wilson, 150-82. Oxford.

Zilliacus, H. 1938. Boktiteln i antik litteratur. Eranos 36: 1-41.

Zimmermann, B. 1992. Dithyrambos. Geschichte einer Gattung. Göttingen. 